\title{
A survey of the higher Stasheff-Tamari orders
}

Jörg Rambau and Victor Reiner

\section{- Preliminary Draft as of October 18, 2011 -}

\begin{abstract}
The Tamari lattice, thought as a poset on the set of triangulations of a convex polygon with $n$ vertices, generalizes to the higher Stasheff-Tamari orders on the set of triangulations of a cyclic $d$-dimensional polytope having $n$ vertices. This survey discusses what is known about these orders, and what one would like to know about them.
\end{abstract}

\section{Introduction}

One often thinks of the Tamari order as a partial order on parenthesizations, or on binary trees. But it can also be taken as an order on triangulations of any $n$-gon whose vertices lie in convex position.

Choosing the vertices of the $n$-gon to lie on a parabola, or 2-dimensional moment curve, lends itself to a beautiful geometric interpretation for the order. This interpretation generalizes to give two closely related orders on the set of triangulations of a cyclic polytope $\mathbf{C}(n, d)$, which is the convex hull of any $n$ points on the $d$-dimensional moment curve.

These orders, called the higher Stasheff-Tamari orders $\operatorname{HST}_{1}(n, d)$ and $\operatorname{HST}_{2}(n, d)$, first appeared roughly 20 years ago in the work of Kapranov and Voevodsky [24, Defn. 3.3], and are somewhat mysterious. Nevertheless, they share many beautiful properties with the Tamari order. Here we survey the work on them by Edelman and Reiner [15], Rambau [31], Reiner [36, §6] Edelman, Rambau and Reiner [14], Thomas [43, 44], and most recently, Oppermann and Thomas [26]. We also discuss

Jörg Rambau

University of Bayreuth, Germany, e-mail: joerg.rambauduni-bayreuth.de

Victor Reiner

University of Minnesota, Minneapolis, USA, e-mail: reiner@math.umn .edu 
work on the closely related Baues problem for subdivisions of cyclic polytopes and zonotopes, as studied by Rambau and Santos [33], Athanasiadis, Rambau and Santos [3], and Athanasiadis [2].

Along the way, we indicate which questions about them remain open.

\section{Cyclic polytopes}

One way to realize the vertices of an $n$-gon in convex position is to pick the vertices as $n$ points with distinct $x$-coordinates on the parametrized parabola $\left\{\left(t, t^{2}\right): t \in \mathbb{R}\right\}$ within $\mathbb{R}^{2}$. More generally, one can define (see [47, Example 0.6]) the $d$-dimensional moment curve in $R^{d}$ as the image of the parametrization

$$
\begin{aligned}
\mathbb{R} \stackrel{v_{d}}{\rightarrow} \mathbb{R}^{d} \\
t \mapsto\left(t, t^{2}, \ldots, t^{d}\right) .
\end{aligned}
$$

Definition 2.1. The $d$-dimensional cyclic polytope with $n$ vertices $\mathbf{C}(n, d)$ is the convex hull of any $n$ points $v_{d}\left(t_{1}\right), \ldots, v_{d}\left(t_{n}\right)$ with distinct $x_{1}$-coordinates

$$
t_{1}<t_{2}<\cdots<t_{n}
$$

We adopt the convention when $d=0$ that these $n$ points are copies of the unique point of $\mathbb{R}^{0}$.

An exercise in Vandermonde determinants and polynomial algebra and inequalities [47. Example 0.6, Theorem 0.7, Exercise 0.8] shows that, no matter how one chooses the $x_{1}$-coordinates in 22 , the polytope $\mathbf{C}(n, d)$ has these combinatorial properties:

- $\mathbf{C}(n, d)$ is a simplicial polytope, meaning that its boundary faces are all simplices,

- $\mathbf{C}(n, d)$ has the same subsets of indices $\left\{i_{0}, i_{1}, \ldots, i_{k}\right\}$ indexing boundary faces $\operatorname{conv}\left\{v_{d}\left(t_{i_{0}}\right), v_{d}\left(t_{i_{1}}\right), \ldots, v_{d}\left(t_{i_{k}}\right)\right\}$, dictated by Gale's evenness criterion, and in particular,

- $\mathbf{C}(n, d)$ is $\left\lfloor\frac{d}{2}\right\rfloor$-neighborly, meaning that every vertex subset of size at most $\frac{d}{2}$ spans a simplex on the boundary.

In light of these properties, it is fair to talk about $\mathbf{C}(n, d)$ and its boundary faces indexed by sets of subscripts $\left\{i_{0}, i_{1}, \ldots, i_{k}\right\}$, without reference to the choice of $x_{1}$-coordinates in (2). In the terminology of oriented matroid theory, the affine point configuration given by the points with homogeneous coordinates $\left\{\left(1, t_{i}, t_{i}^{2}, \ldots, t_{i}^{d}\right)\right\}_{i=1,2, \ldots, n}$ realizes the alternating oriented matroid [9, Cor. 8.2.10], regardless of the choice in (2).

Note also that if one fixes this choice (2), but varies the dimension $d$, then one has canonical projection maps $\pi: C\left(n, d^{\prime}\right) \rightarrow C(n, d)$ for $d^{\prime} \geq d$ by forgetting the 


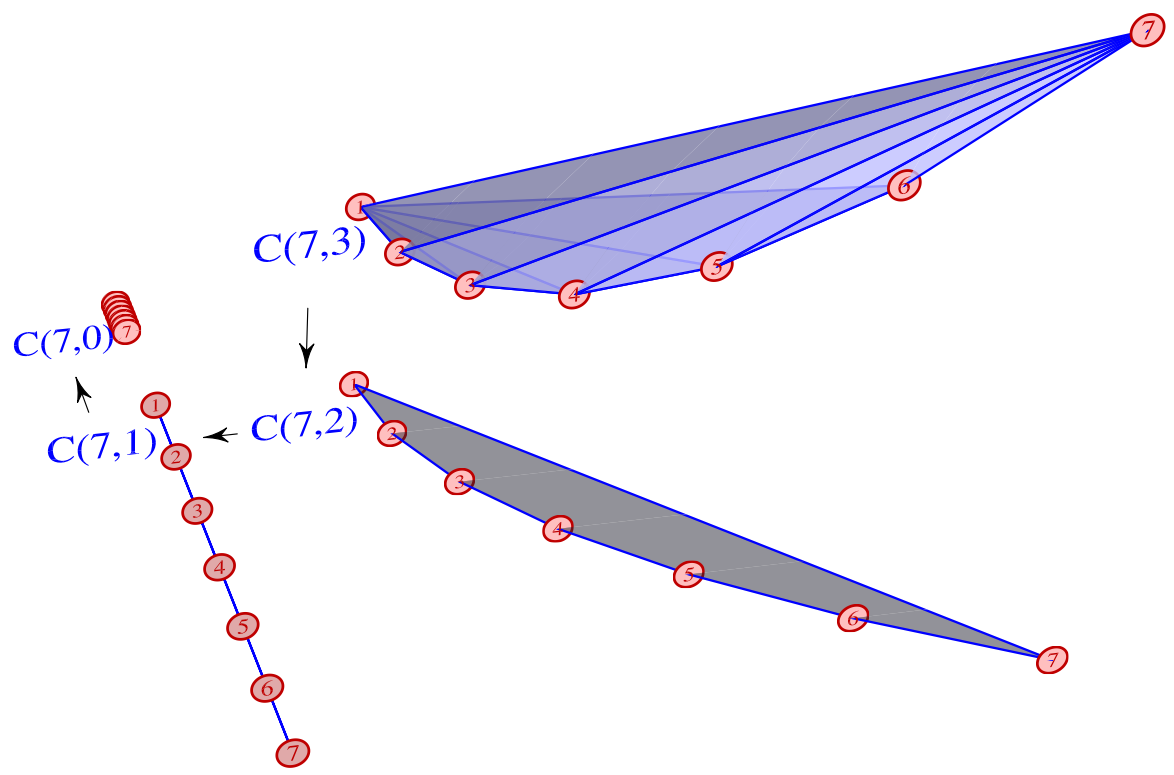

Fig. 1 Cyclic polytopes $\mathbf{C}(7,3), \mathbf{C}(7,2), \mathbf{C}(7,1)$, and $\mathbf{C}(7,0)$ (seven repeated points at the origin) together with the canonical projections forgetting the last coordinate. The bottom triangulation $\hat{0}_{7,2}$ of $\mathbf{C}(7,2)$, discussed in Section 3 is faintly visible as the (obscured) lower facets of $\mathbf{C}(7,3)$.

last $d^{\prime}-d$ coordinates. Figure 1 shows the cyclic polytopes $C(7, d)$ for $d=0,1,2,3$, along with these projection map $\llbracket$.

Because the oriented matroid data for the affine point configuration $\left\{v_{d}\left(t_{i}\right)\right\}_{i=1,2, \ldots, n}$ is independent of the choice (2), it is also well-defined to say when a collection $\mathscr{T}$ of $(d+1)$-element subsets $\left\{i_{1}, i_{2}, \ldots, i_{d+1}\right\}$ indexes the maximal simplices $\operatorname{conv}\left\{v_{d}\left(t_{i_{1}}\right), \ldots, v_{d}\left(t_{i_{d+1}}\right)\right\}$ in a triangulation of the cyclic polytope $\mathbf{C}(n, d)$. For complete discussions of the motivations and technicalities here, see Rambau [31, §2] and DeLoera, Rambau, and Santos [11, Chap. 2].

We will say more about how one encodes or characterizes the collections $\mathscr{T}$ of $(d+1)$-subsets that index triangulations of $\mathbf{C}(n, d)$ in Section 4

\section{The two orders}

The two Stasheff-Tamari orders come from thinking about how a triangulation $\mathscr{T}$ of $\mathbf{C}(n, d)$ induces a section

${ }^{1}$ The astute reader will notice that the point configurations $\mathbf{C}(7,1)$ and $\mathbf{C}(7,0)$ are not really determined by the polytope which is their convex hull. We will tacitly use the term "polytope", even though in certain situations, there is a point configuration in the background which is really part of the data. This becomes even more apparent in the case of cyclic zonotopes discussed in Section 8 We elaborate no further on this here, but refer to [11, Chp. 2] for a technically satisfying setup. 


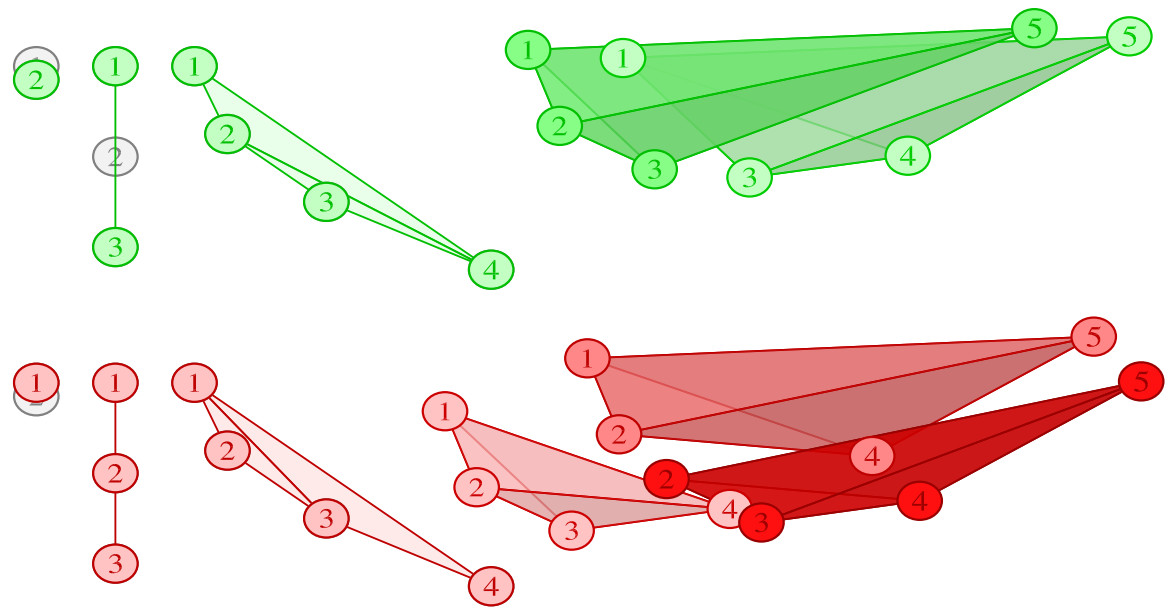

Fig. 2 The two triangulations (green and red) of $\mathbf{C}(d+2, d)$ for small $d$, specifically, $d=0:\{1\}$ versus $\{2\}$ $d=1:\{1,3\}$ versus $\{1,2\},\{2,3\}$ $d=2:\{1,2,4\},\{2,3,4\}$ versus $\{1,2,3\},\{1,3,4\}$ $d=3:\{1,2,3,4\},\{1,2,4,5\},\{2,3,4,5\}$ versus $\{1,2,3,5\},\{1,3,4,5\}$, depicted here in an exploded view: the 3 -simplices are moved slightly apart to clarify how they assemble.

$$
\mathbf{C}(n, d) \stackrel{s_{\mathscr{T}}}{\rightarrow} \mathbf{C}(n, d+1)
$$

of the projection map $\mathbf{C}(n, d+1) \stackrel{\pi}{\rightarrow} \mathbf{C}(n, d)$, defined uniquely by insisting that $s_{\mathscr{T}}$ sends $v_{d}\left(t_{i}\right) \mapsto v_{d+1}\left(t_{i}\right)$, and then extending $s \mathscr{T}$ piecewise-linearly over each simplex in the triangulation $\mathscr{T}$.

From this point of view (and after staring at $\mathbf{C}(n, 3)$ in Figure 1 for a bit), one realizes that the top and bottom elements in the usual Tamari poset correspond to the two canonical triangulations of $\mathbf{C}(n, 2)$ that come from the "upper" and "lower" facets of $\mathbf{C}(n, 3)$. In general, one obtains a canonical upper (resp. lower) triangulation of $\mathbf{C}(n, d)$ by projecting via $\pi: \mathbf{C}(n, d+1) \rightarrow \mathbf{C}(n, d)$ the boundary facets of $\mathbf{C}(n, d+1)$ visible from points with large (resp. small) $x_{d+1}$ coordinate. It is not hard to see that when $n=d+2$, these are the only two triangulations of a cyclic polytope $\mathbf{C}(d+2, d)$; for $d=0,1,2,3$, they are pictured in Figure 2 See also Figure 10 for the $d=3$ case. Explicit descriptions of these canonical upper and lower triangulations for general $d$ may be found in [15, Lemma 2.3].

Definition 3.1. Given two triangulations $\mathscr{T}, \mathscr{T}^{\prime}$ of the cyclic polytyope $\mathbf{C}(n, d)$, say that they are related as $\mathscr{T} \leq_{2} \mathscr{T}^{\prime}$ in the second higher Stasheff-Tamari order $\operatorname{HST}_{2}(n, d)$ if $s_{\mathscr{T}}(x)_{d+1} \leq s_{\mathscr{T}^{\prime}}(x)_{d+1}$ for every point $x$ of $\mathbf{C}(n, d)$, that is, the section $s_{\mathscr{T}}$ lies weakly below the section $s_{\mathscr{T}}$ with respect to their $x_{d+1}$-coordinates.

Definition 3.2. To define the first higher Stasheff-Tamari order $\operatorname{HST}_{1}(n, d)$ on triangulations of $\mathbf{C}(n, d)$, first define when $\mathscr{T}^{\prime}$ is obtained from $\mathscr{T}$ by an upward flip: this means that there exists a $(d+2)$-subset $i_{1}<i_{2}<\cdots<i_{d+2}$ whose convex hull gives a subpolytope $\mathbf{C}(d+2, d)$ of $\mathbf{C}(n, d)$ with the property that $\mathscr{T}, \mathscr{T}^{\prime}$ restrict to 


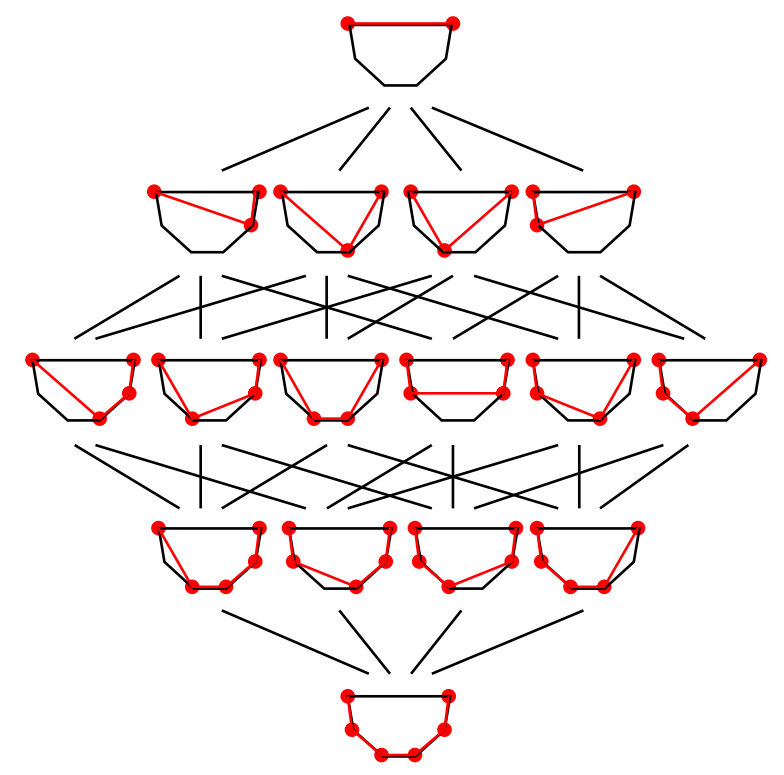

Fig. 3 The (lower!) Stasheff-Tamari orders $\operatorname{HST}_{2}(6,1)=\operatorname{HST}_{1}(6,1)$ on the set of triangulations $\mathscr{T}$ of the line segment $\mathbf{C}(6,1)$. Instead of the triangulation $\mathscr{T}$, its image under the piecewise linear section $s_{\mathscr{T}}: \mathbf{C}(6,1) \rightarrow \mathbf{C}(6,2)$ is depicted in red.

the lower, upper triangulations of this $\mathbf{C}(d+2, d)$, and otherwise $\mathscr{T}, \mathscr{T}^{\prime}$ agree on all of their other simplices not lying in this $\mathbf{C}(d+2, d)$.

Then define $\mathscr{T} \leq_{1} \mathscr{T}^{\prime}$ in $\operatorname{HST}_{1}(n, d)$, if there is a sequence of upward flips starting with $\mathscr{T}$ and ending with $\mathscr{T}^{\prime}$. That is, $\operatorname{HST}_{1}(n, d)$ is the transitive closure of the upward flip relation.

Figure 3 illustrates $\operatorname{HST}_{2}(6,1)$. It should be clear from the definitions and the above discussion that $\leq_{1}$ is a weaker partial order than $\leq_{2}$, and that the lower and upper triangulations of $\mathbf{C}(n, d)$ give the unique minimal $\hat{0}_{n, d}$ and maximal $\hat{1}_{n, d}$ elements of $\operatorname{HST}_{2}(n, d)$. It was left open in [15], and resolved by Rambau affirmatively in [31], that these two triangulations also give unique minimal and maximal elements of $\operatorname{HST}_{1}(n, d)$. In particular, this resolves the question of bistellar connectivity for triangulations of $\mathbf{C}(n, d)$ : any pair of triangulations can be related by a sequence of bistellar flips (see Section 6). It is also closely related to the Generalized Baues Problem for cyclic polytopes, discussed in Section 7 below.

It was shown in [15] that the two orders $\operatorname{HST}_{1}(n, d)$ and $\operatorname{HST}_{2}(n, d)$ are the same for $d=0,1,2,3$, and this is also not hard to check that they are the same when $n-d=1,2,3$. This raises the following question that remains open.

Open Problem 3.3. Are $\operatorname{HST}_{1}(n, d)$ and $\operatorname{HST}_{2}(n, d)$ the same orders?

Historically, the order $\operatorname{HST}_{1}(n, d)$ is the one introduced, in the different terminology of pasting schemes, by Kapranov and Voevodsky [24, Def. 3.3]; the second order $\mathrm{HST}_{2}(n, d)$ was defined in [15, p. 132]. 
The higher Stasheff-Tamari posets for $d=0,1,2$ are familiar objects, as we next explain.

Example 3.4. For $d=0$, the cyclic polytope $\mathbf{C}(n, 0)$ is the unique point of $\mathbb{R}^{0}$, however, it is viewed as a point configuration in which there are $n$ different possible labels $i$ in $\{1,2, \ldots, n\}$ for this point. A triangulation $\mathscr{T}$ of $\mathbf{C}(n, 0)$ is a choice of one of these labels $i$, and an upward flip replaces the label $i$ by the label $i+1$. Thus $\operatorname{HST}_{1}(n, d)$ and $\mathrm{HST}_{2}(n, d)$ both equal the linear order $1<2<\cdots<n$.

Example 3.5. For $d=1$, the cyclic polytope $\mathbf{C}(n, 1)$ is a line segment $\left[t_{1}, t_{n}\right]$ inside $\mathbb{R}^{1}$, however, it is viewed as a point configuration in which there are $n-2$ interior vertices $\left\{t_{2}, t_{3}, \ldots, t_{n-1}\right\}$. Any subset of these interior vertices determines a unique triangulation $\mathscr{T}$ of the line segment $\mathbf{C}(n, 1)$ into smaller segments. A typical upward flip replaces two consecutive smaller segments $\left[t_{i}, t_{j}\right],\left[t_{j}, t_{k}\right]$ having $i<j<k$ with the single segment $\left[t_{i}, t_{k}\right]$, or equivalently, removes $t_{j}$ from the subset of interior vertices used in the triangulation. Thus $\operatorname{HST}_{1}(n, d)$ and $\operatorname{HST}_{2}(n, d)$ are both isomorphic to the Boolean algebra $2^{\left\{t_{2}, t_{3}, \ldots, t_{n-1}\right\}}$. This was illustrated for $n=5$ already in Figure 3 , depicting $\operatorname{HST}_{2}(6,1)=\operatorname{HST}_{1}(6,1)$, which is isomorphic to the Boolean algebra $2^{\left\{t_{2}, t_{3}, t_{4}, t_{5}\right\}}$.

Example 3.6. For $d=2$, as mentioned above, the cyclic polytope $\mathbf{C}(n, 2)$ is a convex $n$-gon. A typical upward flip starts with a triangulated sub-quadrilateral $\mathbf{C}(4,2)$ with four vertices $i<j<k<\ell$ which is triangulated via the two triangles $\{i j k, i k \ell\}$, and replaces it with the same triangulation except for using the two triangles $\{i j \ell, j k \ell\}$ instead. Thus $\operatorname{HST}_{1}(n, 2)$ is equivalent to one of the usual definitions of the Tamari order. It is not completely obvious that $\operatorname{HST}_{1}(n, 2)=\operatorname{HST}_{2}(n, 2)$; a proof appears in [15, Theorem 3.8].

Example 3.7. Figures 4 through 6 show pictures of $\operatorname{HST}_{1}(6,2), \operatorname{HST}_{1}(6,3)$, and $\operatorname{HST}_{1}(7,3)$, respectively, all supported by TOPCOM [32].

The following property, suggested by the previous examples and scrutiny of the accompanying figures, is easily deduced from the definitions.

Proposition 3.8. [15. Prop. 2.11] In both posets $\operatorname{HST}_{1}(n, d), \mathrm{HST}_{2}(n, d)$, reversal of the labelling, that is, the relabelling $1 \mapsto n, 2 \mapsto n-1, \ldots, n \mapsto 1$

- induces a non-trivial poset automorphism for $d$ odd, and

- induces a poset anti-automorphism for d even.

Scrutiny of the examples and figures also suggests the following properties, which are not as obvious, but deduced by Rambau in [31, Cor. 12.(i)].

Proposition 3.9. Given a triangulation $\mathscr{T}$ of $\mathbf{C}(n, d)$, let $|\mathscr{T}|$ denote its number of maximal simplices.

- For d even, $|\mathscr{T}|$ is constant, independent of $\mathscr{T}$, equal to $\left(\begin{array}{c}n-e-1 \\ e\end{array}\right)$ if $d=2 e$.

- For $d$ odd, $|\mathscr{T}|$ takes on all values in the range $\left[\left(\begin{array}{c}n-e-1 \\ e-1\end{array}\right),\left(\begin{array}{c}n-e \\ e\end{array}\right)\right]$ if $d=2 e-1$. In fact, $\operatorname{HST}_{1}(n, d)$ is a ranked poset in which $\mathscr{T}$ has rank $\left(\begin{array}{c}n-e \\ e\end{array}\right)-|\mathscr{T}|$. 




Fig. 4 A picture of $\operatorname{HST}_{1}(6,2)=\mathrm{HST}_{2}(6,2)$, similar to [15. Fig. 4(a)]. Triangulations $\mathscr{T}$ of $\mathbf{C}(6,2)$ are depicted as the images of their corresponding sections $s_{\mathscr{T}}: \mathbf{C}(6,2) \rightarrow \mathbf{C}(6,3)$, viewed from above $\mathbf{C}(6,3)$. Labels $\{j \ell, i k\}$ on covering relations indicate supports of the corresponding flips as follows: the 3 -simplex $\{i, j, k, \ell\}$ with $i<j<k<\ell$ supporting the flip has lower facets $\{i j k, i k \ell\}$, and upper facets $\{i j \ell, j k \ell\}$. 


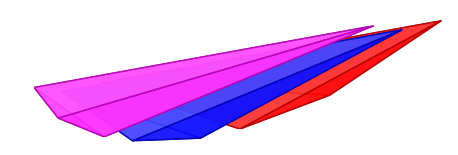

$\{1456,1236,1346\}$

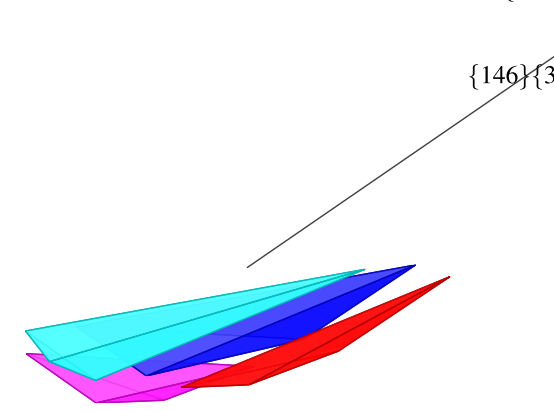

$\{3456,1345,1356,1236\}$

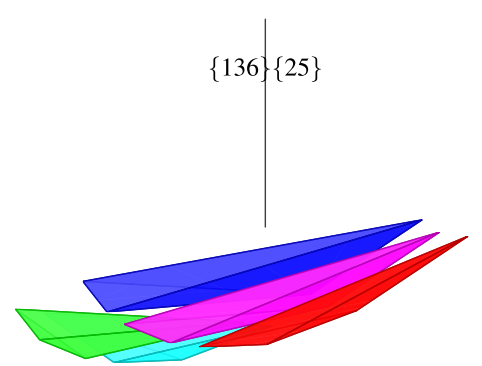<smiles>CCCC</smiles><smiles>CCC[18O]</smiles>

$\{3456,2356,1256,1345,1235\}$

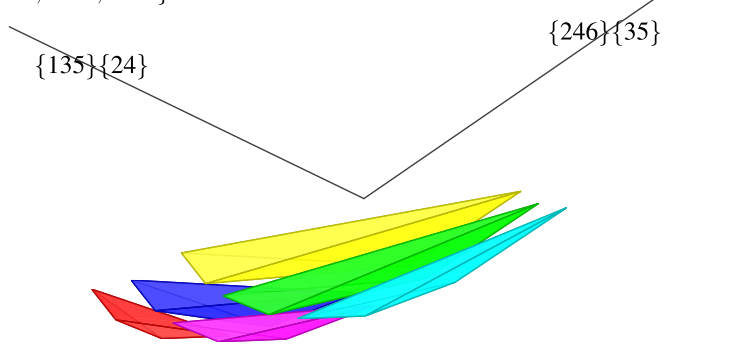

$\{1234,2345,1245,3456,2356,1256\}$

Fig. 5 A picture of $\operatorname{HST}_{1}(6,3)$; the labels of the covering relations indicate the support of the corresponding flip. After reading Theorem 6.6 below, the interested reader may want to find, for each of the 6 triangulations in this figure, at least one maximal chain in Figure 4 which induces it. 


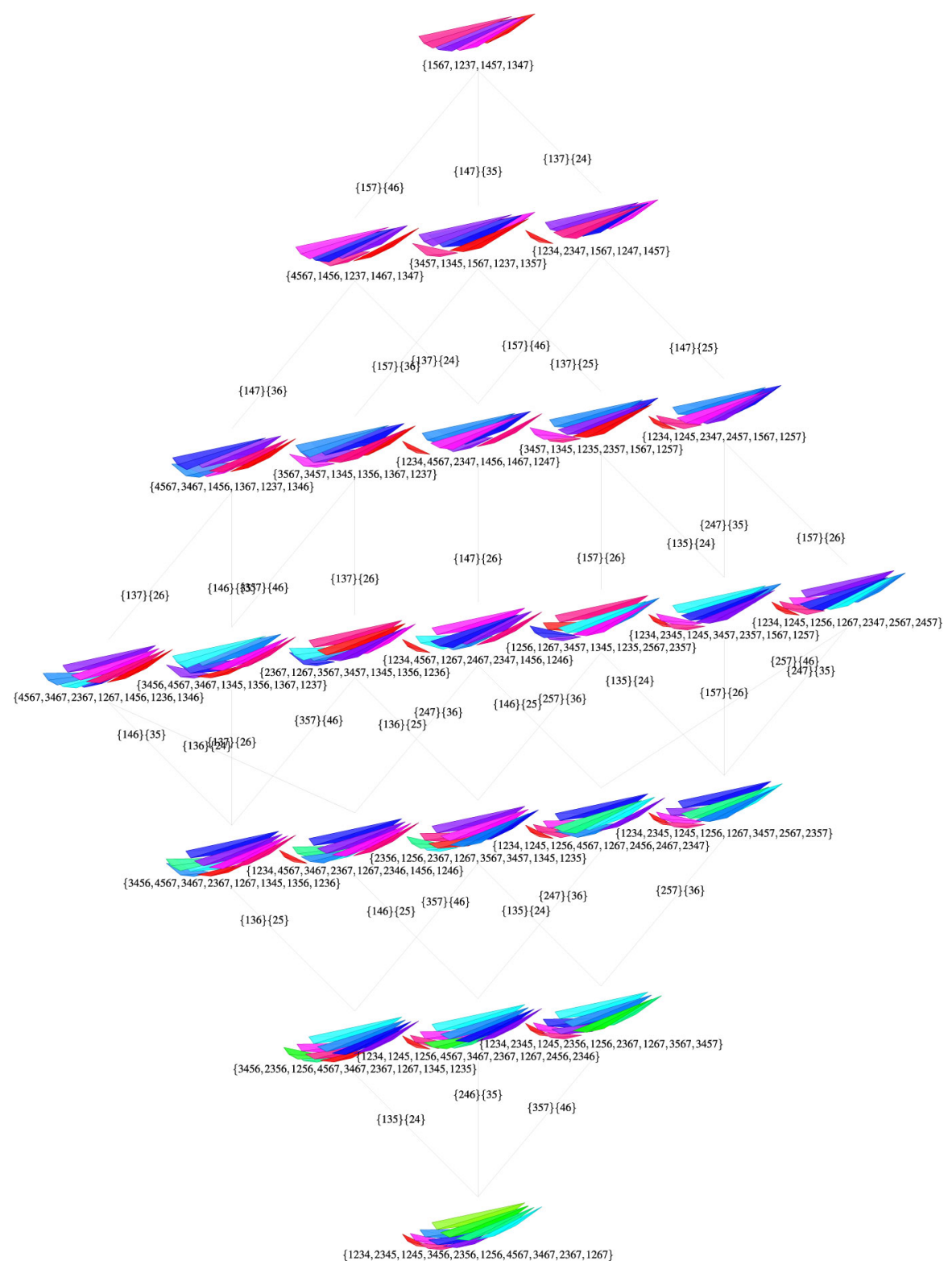

Fig. 6 A picture of $\operatorname{HST}_{1}(7,3)$ (data generated by TOPCOM [32]), similar to [15] Fig. 4(b)]. 


\section{Encodings}

Just as it is sometimes useful to encode elements of the Tamari lattice by other means, such as Huang and Tamari's bracketing vectors [22], it has also proven useful to encode triangulations of $\mathbf{C}(n, d)$ and the Stasheff-Tamari orders in various ways. We discuss three such encodings in the literature, as they appeared in historical order.

\subsection{Submersion sets}

For $k \geq 0$, a subset $\left\{i_{1}, \ldots, i_{k+1}\right\}$ of $\{1,2, \ldots, n\}$, is identified with a $k$-simplex $\sigma=\operatorname{conv}\left\{v_{d}\left(t_{i_{1}}\right), \ldots, v_{d}\left(t_{k+1}\right)\right\}$ inside the cyclic polytope $\mathbf{C}(n, d)$. Denote by $s_{\sigma}$ the unique map $\sigma \rightarrow \mathbb{R}^{d+1}$ that maps its vertices $v_{d}\left(t_{i_{j}}\right) \mapsto v_{d+1}\left(t_{i_{j}}\right)$ for $j=1,2, \ldots, k+1$ and then extends piecewise-linearly over $\sigma$.

Definition 4.1. Given a triangulation $\mathscr{T}$ of $\mathbf{C}(n, d)$, say that $\sigma$ is submerged by $\mathscr{T}$ if $s_{\sigma}(x)_{d+1} \leq s_{\mathscr{T}}(x)_{d+1}$ for all $x$ in $\sigma$. In other words, when one lifts $\sigma$ into $\mathbf{C}(n, d+1)$, it lies weakly below (with respect to $x_{d+1}$-coordinates) the image of the section $s \mathscr{T}$.

Define the $k$-submersion set $\operatorname{sub}_{k}(\mathscr{T})$ to be the collection of subsets $\left\{i_{1}, \ldots, i_{k+1}\right\}$ indexing $k$-simplices $\sigma$ submerged by $\mathscr{T}$.

Proposition 4.2. [15. Prop. 2.15] A triangulation $\mathscr{T}$ of $\mathbf{C}(n, d)$ can be recovered uniquely from its submersion set $\operatorname{sub}_{\left\lceil\frac{d}{2}\right\rceil}(\mathscr{T})$.

Furthermore, $\mathscr{T} \leq_{2} \mathscr{T}^{\prime}$ in $\operatorname{HST}_{2}(n, d)$ if and only if $\operatorname{sub}_{\left\lceil\frac{d}{2}\right\rceil}(\mathscr{T}) \subseteq \operatorname{sub}_{\left\lceil\frac{d}{2}\right\rceil}\left(\mathscr{T}^{\prime}\right)$.

This encoding of $\mathscr{T}$ via sub $\sin _{\left\lceil\frac{d}{2}\right\rceil}(\mathscr{T})$ is used mainly in [15] for $d \leq 3$. There it is explained how to read off $\operatorname{sub}_{\left\lceil\frac{d}{2}\right\rceil}(\mathscr{T})$ from the $d$-simplices of $\mathscr{T}$, and the subsets which can appear as $\operatorname{sub}_{\left\lceil\frac{d}{2}\right\rceil}(\mathscr{T})$ are characterized as follows.

Proposition 4.3. [15. Props. 3.3 and 4.2] For $d=2$, a collection $I=\{i j\} \subset$ $\{1,2, \ldots, n\}$ has $I=\operatorname{sub}_{1}(\mathscr{T})$ for some triangulation of $\mathbf{C}(n, 2)$ if and only if

- I contains every boundary edge of $\mathbf{C}(n, 2)$.

- Assume ik is in I. If $i<j<k$, then $i j$ is also in $I$.

- If $i k, j \ell$ are both in $I$, with $i<j<k<\ell$, then il is also in $I$.

For $d=3$, a collection $I=\{i j k\} \subset\{1,2, \ldots, n\}$ has $I=\operatorname{sub}_{2}(\mathscr{T})$ for some triangulation of $\mathbf{C}(n, 3)$ if and only if

- I contains every boundary triangle of $\mathbf{C}(n, 3)$.

- Assume ijk is in I. If $j<k^{\prime}<k$, then $i j k^{\prime}$ is also in I. If $i<i^{\prime}<j$, then $i^{\prime} j k$ is also in $I$.

- If $i j k, a b c$ are in I, with $a<i<b<j<c<k$, then abk, ajk are also in I. 


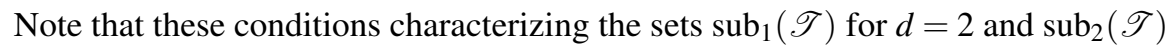
for $d=3$ are closure conditions, and hence they are preserved when one intersects sets. This immediately implies that the second Stasheff-Tamari order $\operatorname{HST}_{2}(n, d)$ is a meet semilattice for $d \leq 3$, with meet operation given by intersecting these sets. Since it has the unique maximal element $\hat{1}_{n, d}$, one immediately deduces the following.

Theorem 4.4. [15. Thms. 3.6 and 4.9] For $d \leq 3$, the higher Stasheff-Tamari order $\operatorname{HST}_{2}(n, d)$ is a lattice.

With some work, these encodings can also be used to show the following previously mentioned result.

Proposition 4.5. [15. Thms. 3.8 and 4.10] For $d \leq 3$, the two Stasheff-Tamari orders $\operatorname{HST}_{1}(n, d), \operatorname{HST}_{2}(n, d)$ are the same.

These encoding also have consequences for the homotopy types of intervals and Möbius functions $\mu(x, y)$ in the orders for $d \leq 3$, to be discussed in Section 5 below.

\subsection{Snug rectangles}

In [43], Thomas presents an amazingly simple encoding of the triangulations $\mathscr{T}$ of $\mathbf{C}(n, d)$, and an accompanying reformulation of the order $\mathrm{HST}_{2}(n, d)$.

Definition 4.6. Let $L(n, d)$ denote the set of all strictly increasing integer sequences $\left(a_{1}, a_{2}, \ldots, a_{d}\right)$ of length $d$ with $1 \leq a_{i} \leq n$. For each $d$-simplex, indexed by the $(d+1)$-subset $i_{1}<i_{2}<\cdots<i_{d+1}$, appearing in $\mathscr{T}$, associate the subset of $L(n, d)$ (called a snug rectangle) which is the following $d$-fold Cartesian product:

$$
\left[i_{1}, i_{2}-1\right] \times\left[i_{2}, i_{3}-1\right] \times \cdots \times\left[i_{d}, i_{d+1}-1\right] .
$$

Given the triangulation $\mathscr{T}$ and its various snug rectangles, let

$$
\begin{aligned}
U_{\mathscr{T}}:=\{ & \left(a_{1}, a_{2}, \ldots, a_{d-1}, a_{d}\right) \in L(n, d): \\
& \left(a_{1}, a_{2}, \ldots, a_{d-1}, a_{d}\right),\left(a_{1}, a_{2}, \ldots, a_{d-1}, a_{d}-1\right) \text { lie in } \\
& \text { the same snug rectangle of } \mathscr{T}\} .
\end{aligned}
$$

Theorem 4.7. [43. Theorems 1.1, 1.2] The map sending a triangulation $\mathscr{T}$ of $\mathbf{C}(n, d)$ to its collection of snug rectangles is a bijection between all triangulations of $\mathbf{C}(n, d)$ and all decompositions of $L(n, d)$ into snug rectangles.

Furthermore, $\mathscr{T} \leq_{2} \mathscr{T}^{\prime}$ in $\operatorname{HST}_{2}(n, d)$ if and only if $U_{\mathscr{T}} \subseteq U_{\mathscr{T}^{\prime}}$.

Thomas goes on to exhibit a natural poset embedding

$$
\operatorname{HST}_{2}(n, d) \hookrightarrow \prod_{j=d}^{n-1} \operatorname{HST}_{2}(j, d-2)
$$




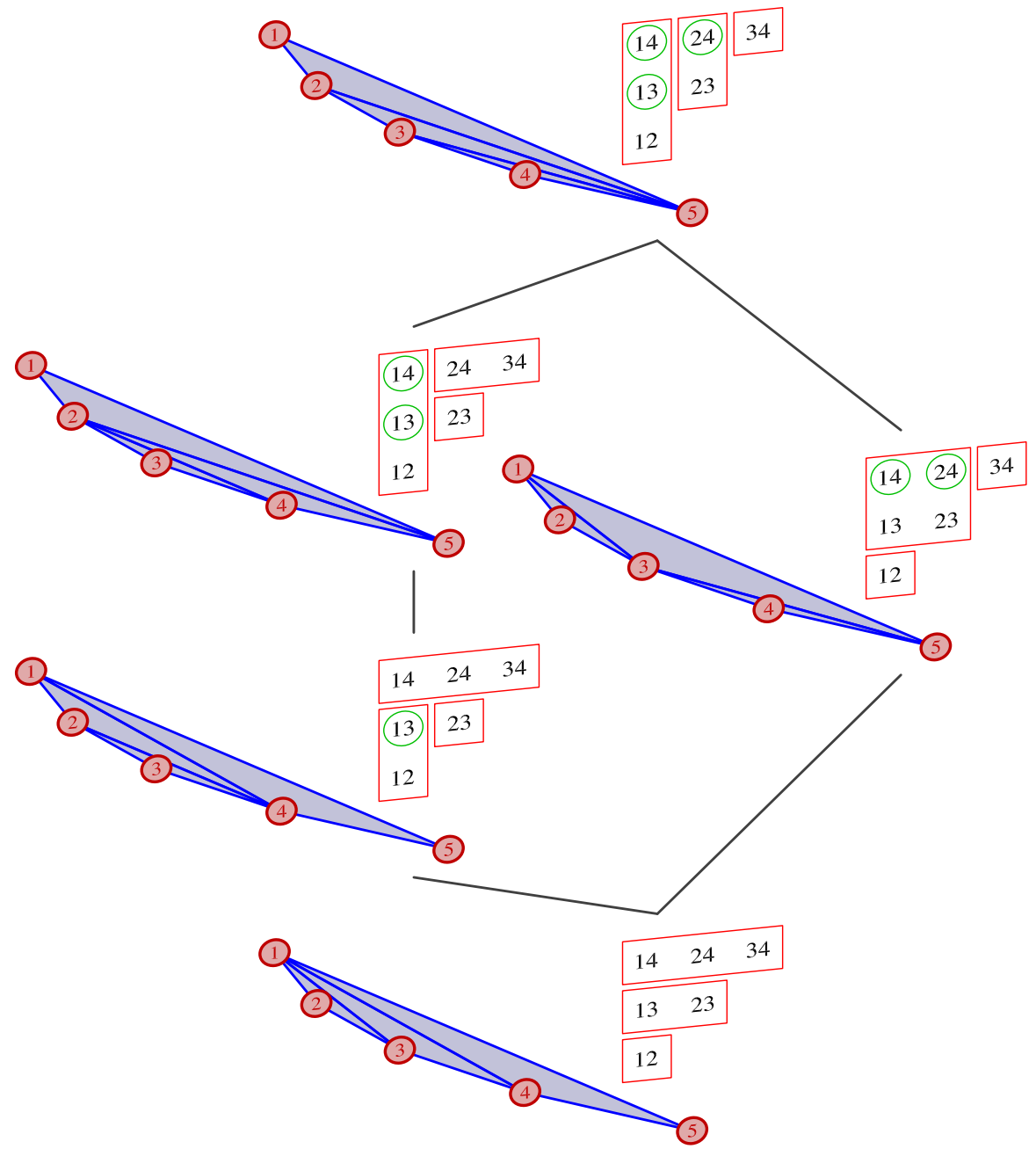

Fig. 7 Triangulations $\mathscr{T}$ of $\mathbf{C}(5,2)$ with their snug rectangle encodings (red), and the points of $U_{\mathscr{T}}$ circled green within the rectangles.

which for $d=2$ turns into Huang and Tamari's bracket vector encoding [22]. By iterating this poset embedding, he improves the upper bounds that had been given in [15] on the order dimension $\operatorname{dim} \operatorname{HST}_{2}(n, d)$, that is, the smallest $N$ for which $\operatorname{HST}_{2}(n, d)$ has a poset embedding into a product of $N$ linear orders. Furthermore, he gets an exact calculation for the 2-dimension $\operatorname{dim}_{2} \operatorname{HST}_{2}(n, d)$, that is, the smallest $N$ for which $\operatorname{HST}_{2}(n, d)$ has a poset embedding into a Boolean algebra $2^{N}$ of rank $N$.

Theorem 4.8. [43. Theorem 6.1] The higher Stasheff-Tamari order $\operatorname{HST}_{2}(n, d)$ has

- for $d=2 e+1$ odd, 


$$
\operatorname{dim}_{H_{S T}}(n, d) \leq \operatorname{dim}_{2} \operatorname{HST}_{2}(n, d)=\left(\begin{array}{c}
n-e-2 \\
e+1
\end{array}\right) \text {, and }
$$

- for $d=2 e$ even,

$$
\begin{aligned}
\operatorname{dim} H_{S T}(n, d) & =\left(\begin{array}{c}
n-e-2 \\
e
\end{array}\right), \\
\operatorname{dim}_{2} \operatorname{HST}_{2}(n, d) & =\left(\begin{array}{c}
n-e-1 \\
e+1
\end{array}\right) .
\end{aligned}
$$

\subsection{Non-interlacing separated $\frac{d}{2}$-faces}

Oppermann and Thomas [26] recently uncovered a fascinating connection between the representation theory of certain algebras and triangulations of the evendimensional cyclic polytopes $\mathbf{C}(m, d)$ with $d=2 e$. We will not do justice to their results here and refer the interested reader to their paper for more details and precise statements.

Very roughly, they give two generalizations to all even $d$ of the following algebraic results for $d=2$ : when one considers the path algebra of the linearly oriented type $A_{n}$ quiver, the set of indecomposables in the module category which are not simultaneously projective and injective (resp. the set of all indecomposables in the cluster category) can be identified with the internal diagonals of $\mathbf{C}(n+2,2)$ (resp. of $\mathbf{C}(n+3,2))$. Furthermore, this can be done in such a way that basic tilting modules correspond to triangulations, and mutations correspond to diagonal flips. For more on this, see the references in Oppermann and Thomas [26], as well as in Thomas's survey [45] in this volume.

In their work, the role played by the internal diagonals of a triangulation $\mathscr{T}$ of $\mathbf{C}(m, 2)$ is played by the nonboundary $e$-dimensional faces contained in a triangulation $\mathscr{T}$ of $\mathbf{C}(m, 2 e)$. They begin with an old observation of Dey [13] that for any $d$, a triangulation $\mathscr{T}$ of any $d$-dimensional polytope is completely determined by the $\left\lfloor\frac{d}{2}\right\rfloor$-dimensional faces that it contains.

In the special case of cyclic polytopes $\mathbf{C}(m, 2 e)$, these collections of $e$-faces have a convenient characterization.

Definition 4.9. Say that an $e$-face with vertex set $i_{1}<i_{2}<\cdots<i_{e+1}$ is separated if $i_{\ell+1}-i_{\ell}>1$ for all $0 \leq \ell \leq e$. Say that two such separated $e$-faces

$$
i_{1}<i_{2}<\cdots<i_{e+1} \text { and } j_{1}<j_{2}<\cdots<j_{e+1}
$$

intertwine if either

$$
i_{1}<j_{1}<i_{2}<j_{2}<\cdots<i_{e+1}<j_{e+1}
$$

or if the same holds reversing the roles of $i$ 's and $j$ 's. 
When $d=2$ and $e=1$, it is not hard to see that the separated $e$-faces of $\mathbf{C}(m, 2)$ correspond to the internal diagonals as well as the "upper" boundary edge $\{1, m\}$, and that the collections of $m-2$ separated $e$-simplices which are pairwise nonintertwining are exactly the sets of internal diagonals of triangulations $\mathbf{C}(m, 2)$, combined with $\{1, m\}$. Oppermann and Thomas generalize this as follows.

Theorem 4.10. [26] Theorems 2.3 and 2.4] Given a triangulation $\mathscr{T}$ of $\mathbf{C}(m, 2 e)$, consider the collections of all of its separated e-simplices, or equivalently, all of its e-simplices that do not lie within the lower boundary of $\mathbf{C}(m, 2 e)$.

Then these are exactly the collections of $\left(\begin{array}{c}m-e-1 \\ e\end{array}\right)$ separated and pairwise nonintertwining e-simplices inside $\mathbf{C}(m, 2 e)$.

They go on to use this characterization in their study of certain categories derived from the module category of the $(e-1)$-fold higher Auslander algebra $A_{n}^{e}$ of the linearly oriented type $A_{n}$ quiver. For $e=1$, this algebra $A_{n}^{e}$ is simply the path algebra of the quiver discussed earlier in this section. They obtain generalizations of the above algebraic statements for $e=1$, by identifying [26, Theorems 1.1, 1.2] the internal $e$-simplices in $\mathbf{C}(n+2 e, 2 e)$ (resp. $\mathbf{C}(n+2 e+1,2 e))$ with certain kinds of indecomposable objects in two different categories constructed from $A_{n}^{e}$-modules. Furthermore, they do this in such a way that, in each case, basic tilting modules correspond to triangulations, and the appropriate analogues of mutation correspond to bistellar flips [26, Theorems 4.4, 6.4].

As crucial tools in their proofs, not only do they use the above encoding of triangulations, but also the result of Rambau [31] mentioned in Section 3; all triangulations of $\mathbf{C}(m, d)$ are connected by a sequence of bistellar flips.

\section{Lattice property, homotopy types and Möbius function}

Theorem 4.4 showed that for $d \leq 3$, the two higher Stasheff-Tamari orders $\operatorname{HST}_{1}(n, d)$ and $\operatorname{HST}_{2}(n, d)$ coincide, and both are lattices. Although it was conjectured there that they remain lattices for all $d$, counterexamples were later found by computer search showing that the lattice property fails, at least for $\operatorname{HST}_{2}(n, d)$, when $(n, d)=(9,4)$ and $(10,5)$; see [14, §7].

On the other hand, for $d \leq 3$, the two coinciding Stasheff-Tamari lattices $\operatorname{HST}_{1}(n, d)$ and $\operatorname{HST}_{2}(n, d)$ enjoy the following pleasant property, which is checked easily for $d \leq 1$, proven for $d=2$ by Pallo [28, Lemma 4.1], and proven for $d=3$ in [15, Theorem 4.11].

Theorem 5.1. Let $d \leq 3$. For an interval $[x, y]$ in the lattices $\operatorname{HST}_{1}(n, d)=$ $\operatorname{HST}_{2}(n, d)$, let $\left\{z_{1}, z_{2}, \ldots, z_{c}\right\}$ be its set of coatoms, that is the elements $z_{i} \geq x$ which are covered by $y$. Then distinct subsets of $\left\{z_{1}, z_{2}, \ldots, z_{c}\right\}$ have distinct meets.

In particular,

- if $z_{1} \wedge \cdots \wedge z_{c}=x$, then the open interval $(x, y)$ is homotopy equivalent to a $(c-2)$-dimensional sphere, and the Möbius function $\mu(x, y)=(-1)^{c}$, 
- if $z_{1} \wedge \cdots \wedge z_{c}>x$, then $(x, y)$ is contractible and $\mu(x, y)=0$.

For dimensions $d>3$, this homotopy type issue is not yet resolved for all intervals, but it is known for the improper open interval $\left(\hat{0}_{n, d}, \hat{1}_{n, d}\right)$, that is, the proper part of the posets, and the answer for all intervals is conjectured, as we next discuss.

Firstly, the useful tool of Rambau's suspension lemma for bounded posets [30], developed to handle the homotopy of the proper parts of the higher Bruhat orders, similarly allowed Edelman, Rambau and Reiner [14, Theorem 1.1] to prove the following.

Theorem 5.2. [14 Theorem 1.1] For $n>d+1$, the proper parts of both posets $\operatorname{HST}_{1}(n, d)$ and $\operatorname{HST}_{2}(n, d)$ are homotopy equivalent to $(n-d-3)$-dimensional spheres 2

Next, when considering intervals $[x, y]$ in $\operatorname{HST}_{2}(n, d)$, an exact conjecture on their homotopy type was formulated in [14]. For this one must introduce the notion of polyhedral subdivisions $\mathscr{S}$ of $\mathbf{C}(n, d)$ (or any point configuration), which are more general than triangulations; we will return to this notion when discussing secondary polytopes in Sections 6.2 and 7.1

Informally, such a subdivision $\mathscr{S}$ is a decomposition of $\mathbf{C}(n, d)$ into subpolytopes $\left\{P_{i}\right\}_{i \in I}$, with these properties:

- each subpolytope $P_{i}$ has vertex set which is a subset of the vertices of $\mathbf{C}(n, d)$, and

- each pair $P_{i}, P_{j}$ of subpolytopes has pairwise intersection $P_{i} \cap P_{j}$ equal to a face (possibly empty) common to both.

There is an obvious notion for when one such subdivision refines another. Having fixed a particular subdivision $\mathscr{S}$ of $\mathbf{C}(n, d)$, when one considers the collection of all triangulations $\mathscr{T}$ that refine it, it is not hard to see that they form an interval $\left[x_{\mathscr{S}}, y_{\mathscr{S}}\right]$ in $\operatorname{HST}_{2}(n, d)$ (or in $\operatorname{HST}_{1}(n, d)$ ). Specifically, if $\mathscr{S}$ has the subpolytope $P_{i}$ isomorphic to $\mathbf{C}\left(n_{i}, d\right)$, then $x_{\mathscr{S}}$ (resp. $y_{\mathscr{S}}$ ) triangulates $P_{i}$ according to the triangulation $\hat{0}_{n_{i}, d}$ (resp. $\left.\hat{1}_{n_{i}, d}\right)$ of $P_{i}$. It is also not hard to see that the closed interval $\left[x_{\mathscr{S}}, y_{\mathscr{S}}\right]$ will be poset-isomorphic to the Cartesian product $\prod_{i \in I} \operatorname{HST}_{2}\left(n_{i}, d\right)$. Hence its proper part, the open interval $\left(x_{\mathscr{S}}, y_{\mathscr{S}}\right)$ will have the homotopy type of a sphere of dimension $-2+\sum_{i \in I}\left(n_{i}-d_{i}-1\right)$, combining Theorem 5.2 with a lemma of Walker [8, eqn (9.8)]: the proper part of the Cartesian product $P_{1} \times P_{2}$ of two bounded posets $P_{1}, P_{2}$ is homeomorphic to the suspension of the join of their proper parts.

Open Problem 5.3. [14] Conjecture 7.1]. Prove that the noncontractible open intervals in $\mathrm{HST}_{2}(n, d)$ are exactly the $\left(x_{\mathscr{S}}, y_{\mathscr{S}}\right)$ coming from subdivisions $\mathscr{S}$ of $\mathbf{C}(n, d)$. In particular, the Möbius function of $\mathrm{HST}_{2}(n, d)$ only takes on values in $\{0, \pm 1\}$.

These assertions are well-known for $d \leq 2$; in the case $d=2$, they assert that the noncontractible intervals in the Tamari lattice $\mathrm{HST}_{2}(n, 2)$ are exactly the coatomic or

\footnotetext{
${ }^{2}$ For $n=d+2$, we are using a standard combinatorial convention: the proper part of a poset having only two elements $\{x, y\}$ with $x<y$ is the simplicial complex $\{\varnothing\}$ having only the (-1)-dimensional empty face and no other faces, and considered to triangulate a $(-1)$-dimensional sphere.
} 


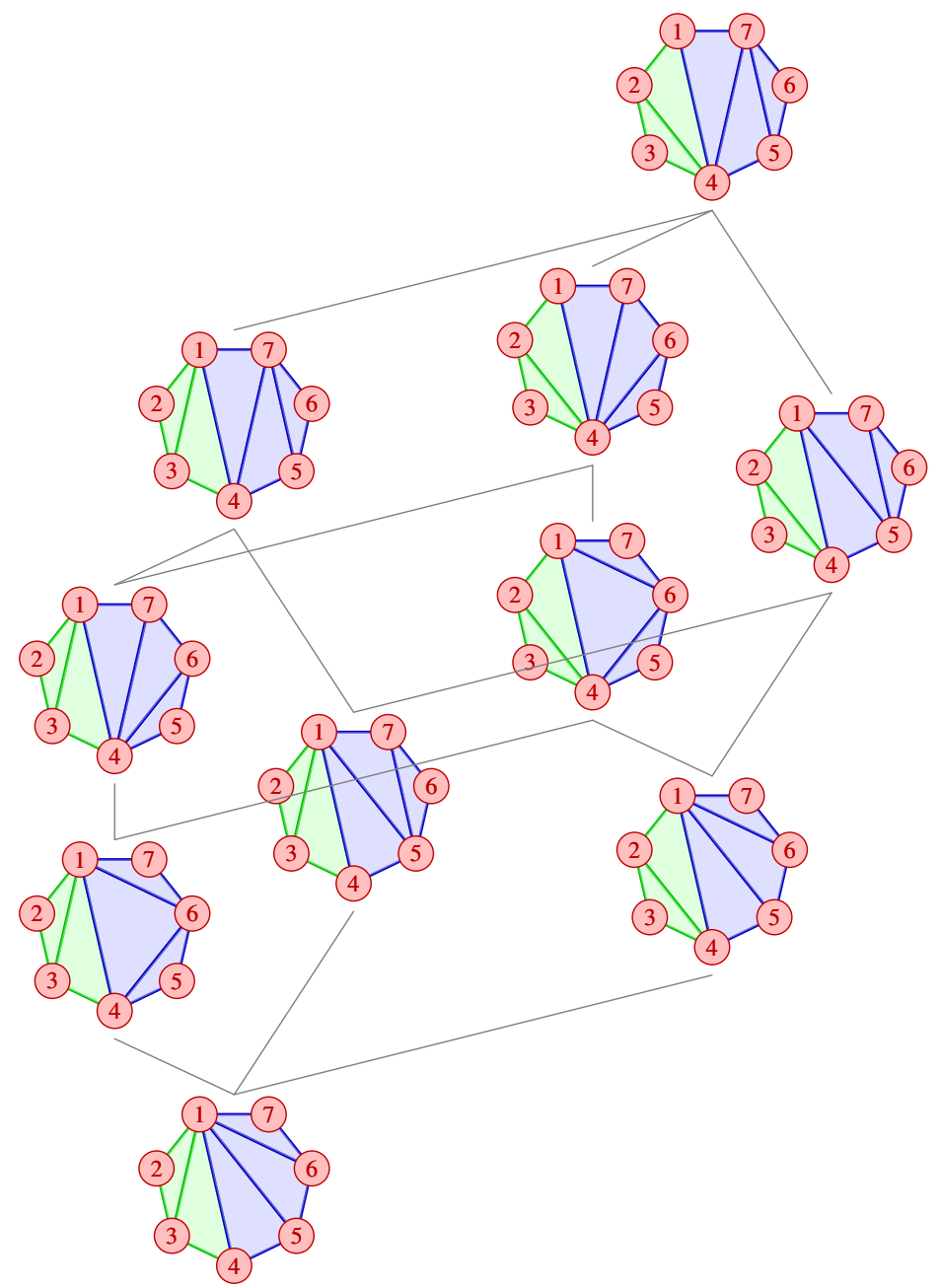

Fig. 8 A subdivision $\mathscr{S}$ of $\mathbf{C}(7,2)$ into a green quadrangle and a blue pentagon, along with its facial interval $\left[x_{\mathscr{S}}, y_{\mathscr{S}}\right] \cong \mathrm{HST}_{2}(4,2) \times \operatorname{HST}_{2}(5,2)$ in $\operatorname{HST}_{2}(7,2)$. The open interval $\left(x_{\mathscr{S}}, y_{\mathscr{S}}\right)$ is homotopy equivalent to a 1-sphere (circle). The heptagon $\mathbf{C}(7,2)$ is depicted with respect to coordinates on the Caratheodory curve, rather than the moment curve, for better visibility of triangles.

facial intervals $[x, y]$, that is, those in which $x=x_{\mathscr{S}}, y=y_{\mathscr{S}}$ are the minimum and maximum elements lying on a particular face of the associahedron, indexed by a polygonal subdivision $\mathscr{S}$ of the $n$-gon $\mathbf{C}(n, 2)$; see Huguet and Tamari [23], and Pallo [28]. Figure 8 shows an example of such an interval $\left[x_{\mathscr{S}}, y_{\mathscr{S}}\right]$ within $\mathrm{HST}_{2}(7,2)$, with in this case an isomorphism $\left[x_{\mathscr{S}}, y_{\mathscr{S}}\right] \cong \operatorname{HST}_{2}(4,2) \times \operatorname{HST}_{2}(5,2)$. 


\section{Connection to Flip Graph Connectivity}

The Hasse diagram for the higher Stasheff-Tamari order $\operatorname{HST}_{1}(n, d)$, considered as an undirected graph, is a special case of an important concept from discrete and computational geometry, which we discuss here: the flip graph of all triangulations and (bistellar) flips for an arbitrary affine point configuration $\mathbf{A}$ in $\mathbb{R}^{d}$.

\subsection{Bistellar flips}

Recall that an edge in the Hasse diagram for $\operatorname{HST}_{1}(n, d)$ corresponds to two triangulations $\mathscr{T}, \mathscr{T}^{\prime}$ of $\mathbf{A}=\mathbf{C}(n, d)$ that share almost all of the same simplices except that they restrict to the two different possible triangulations (upper and lower) of the convex hull of a certain subset $\mathbf{A}^{\prime}=\mathbf{C}(d+2, d)$ of cardinality $d+2$.

It remains true generally that for $d+2$ points $\mathbf{A}^{\prime}$ in $\mathbb{R}^{d}$, there will be exactly two triangulations of their convex hull, using only vertices in $\mathbf{A}^{\prime}$. It even remains true that these two triangulations will again be the set of "upper" and "lower" facets for some lifting of the points $\mathbf{A}^{\prime}$ in $\mathbb{R}^{d}$ to the vertices of a $(d+1)$-simplex in $\mathbb{R}^{d+1}$, but the combinatorics of these two triangulations will depend upon the signs in the unique affine dependence (up to scaling) among these points, or the oriented matroid of the affine point configuration $\mathbf{A}^{\prime}$; see again [11, §2.4].

Definition 6.1. Two triangulations $\mathscr{T}, \mathscr{T}^{\prime}$ of the convex hull of an affine point configuration $\mathbf{A}$ in $\mathbb{R}^{d}$ using only vertices in $\mathbf{A}$, are said to differ by a (d-dimensional) bistellar flip if they share almost all of the same simplices, but restrict to the two possible triangulations of the convex hull of some $d+2$ element subset $\mathbf{A}^{\prime} \subset \mathbf{A}$.

More generally than the $d$-dimensional bistellar flips, one also allows lowerdimensional bistellar flips between two triangulations $\mathscr{T}, \mathscr{T}^{\prime}$, involving a subset $\mathbf{A}^{\prime} \subset \mathbf{A}$ of cardinality $e+2$ whose affine span is $e$-dimensional; see again [11, §2.4] for the precise definitions. Figure 9 illustrates some of the variety of flips possible already for points $\mathbf{A}$ in $\mathbb{R}^{2}$, with the rightmost example being lower-dimensional flip. Although the variety of possible types of flips grows in higher dimensions (see Figure 10 for one example), when $\mathbf{A}$ in $\mathbb{R}^{d}$ is in general position (no $d+1$ of its points lie on an affine hyperplane of $\mathbb{R}^{d}$ ), the flips are local modifications, that affect at most $d+1$ simplices on $d+2$ points in a triangulation. Thus, flips are important in computational geometry ( $d=2$ or $d=3$, mostly!) as a means to improve triangulations by local modifications (see [17] for just one example or [16] and [10, Chps. 3 and 9] for the low-dimensional viewpoint of Computational Geometry). In non-general position, flips can become quite large modifications. (See also [11, Chp. 8] for a more detailed discussion on algorithmic issues in general dimension).

We should warn the reader that there is a closely related notion of bistellar flip in the literature, which is not quite the same: bistellar equivalences for triangulations of PL-manifolds, as in the work of Pachner [27]. There one does not insist that the manifolds have a fixed embedding into space nor that the vertices in the triangulation 

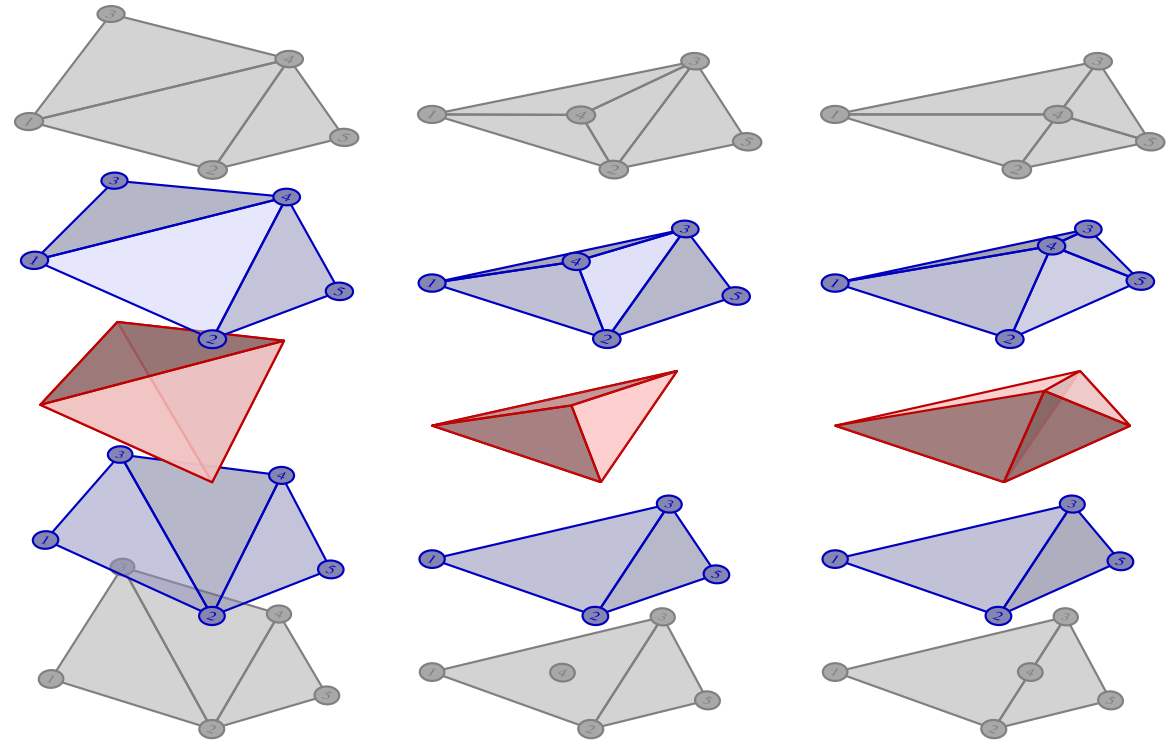

Fig. 9 An edge flip and a vertex flip in dimension two (grey), whose combinatorics can be represented topologically by pushing a surface in dimension three (blue) through a tetrahedron (red) all the way from the lower facets to the upper facets. The rightmost figure is a lower-dimensional flip, adding vertex 4 in the middle of edge 23 (grey): its combinatorics can represented topologically by pushing a surface in dimension three (blue) through a vertical triangle (=2-simplex!) linked to two vertices (red).

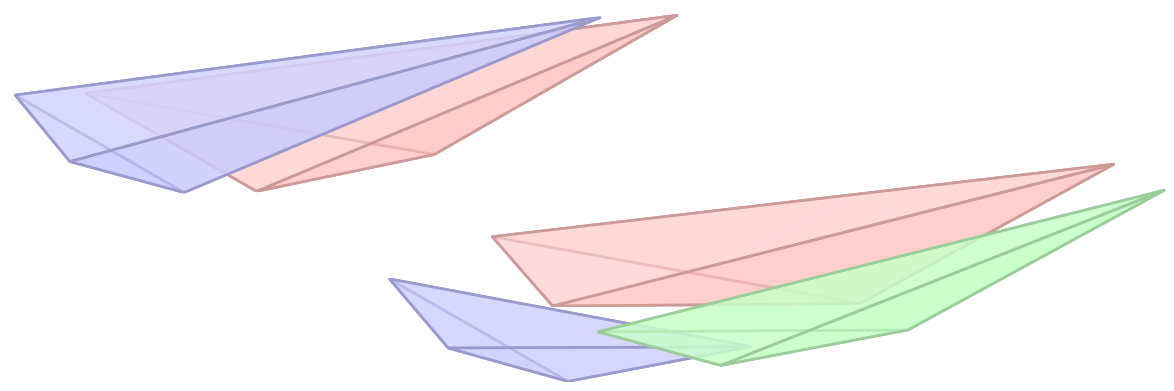

Fig. 10 In dimension three, general position flips will change the number of simplices, as in $\mathbf{C}(5,3)$ depicted here, which has exactly these two triangulations (exploded view). Compare with the discussion of (2,3)-Pachner moves in the survey by Stasheff in this volume [41, §4.2].

have fixed coordinates. In contrast, triangulations in our context have vertices coming from the point set $\mathbf{A}$, with fixed coordinates in $\mathbb{R}^{d}$. 


\subsection{The flip connectivity question}

In discrete and computational geometry, one would like to use bistellar flips to explore the set of all triangulations of $\mathbf{A}$, or to get to any triangulation (for example, a special desired one) from any other triangulation (for example, an obvious one, such as the popular Delaunay triangulation [11, $\S 2.2 .2]$. This motivates the following definition and question.

Definition 6.2. Given an affine point configuration $\mathbf{A}$ in $\mathbb{R}^{d}$, its flip graph $\mathscr{G}_{\text {tri }}(\mathbf{A})$ has vertex set indexed by the triangulations $\mathscr{T}$ of the convex hull of $\mathbf{A}$ using only vertices in $\mathbf{A}$, and edges between pairs of triangulations $\mathscr{T}, \mathscr{T}^{\prime}$ whenever they differ by a bistellar flip.

Question 6.3. Given an affine point configuration $\mathbf{A}$ in $\mathbb{R}^{d}$, is $\mathscr{G}_{\text {tri }}(\mathbf{A})$ connected?

When either $d \leq 2$, or $|\mathbf{A}|-d \leq 3$, it is not hard to prove that the answer is "Yes". For higher dimensions $d$ and point configurations $\mathbf{A}$, this question tantalized researchers for quite some time until resolved negatively by Santos, first in [38], where he found a counter-example with $d=6$, double-checked by computer-calculations with TOPCOM [32]. Later Santos [39] produced another counter-example $d=5$ and in general position, which can be turned into convex-position examples by a standard construction, the Lawrence construction [11, §5.5].

Theorem 6.4. [39] Theorem 1] There is a 5-dimensional polytope with vertex set $\mathbf{A}$ of cardinality 26 for which the flip graph $\mathscr{G}_{\text {tri }}(\mathbf{A})$ is disconnected.

This should be compared with the positive results of Gelfand, Kapranov and Zelevinsky on secondary polytopes [19]. They distinguish a particularly well-behaved subgraph of $\mathscr{G}_{\text {tri }}(\mathbf{A})$, which is not only connected, but even $(|A|-d-1)$-vertexconnected in the graph-theoretic sense, because it gives the 1-skeleton (vertices and edges) of the $(|A|-d-1)$-dimensional secondary polytope. This subgraph consists of the regular triangulations or coherent triangulations (and the regular flips or coherent flips between them), namely those that arise as projections of lower facets of a lifting of the point configuration.

\subsection{The flip graph of a cyclic polytope}

Returning to cyclic polytopes $\mathbf{C}(n, d)$, it is known and not hard to see that for $d=2$, all triangulations are regular/coherent. This corresponds to the fact that the Hasse diagram of the Tamari order is the 1-skeleton of the Stasheff polytope or associahedron, which is the secondary polytope for the point configuration $\mathbf{C}(n, 2)$. However, for any fixed $d \geq 3$, one can show that, asymptotically in $n$, most triangulations of $\mathbf{C}(n, d)$ are not regular/coherent, [11, $\S 6.1]$, which raises that question of connectivity for their flip graphs.

Theorem 6.5. [31. Thm. 1.1, Cor. 1.2]. The first higher Stasheff-Tamari order $\operatorname{HST}_{1}(n, d)$ is bounded, with the same bottom $\hat{0}_{n, d}$ and top $\hat{1}_{n, d}$ triangulations as the 


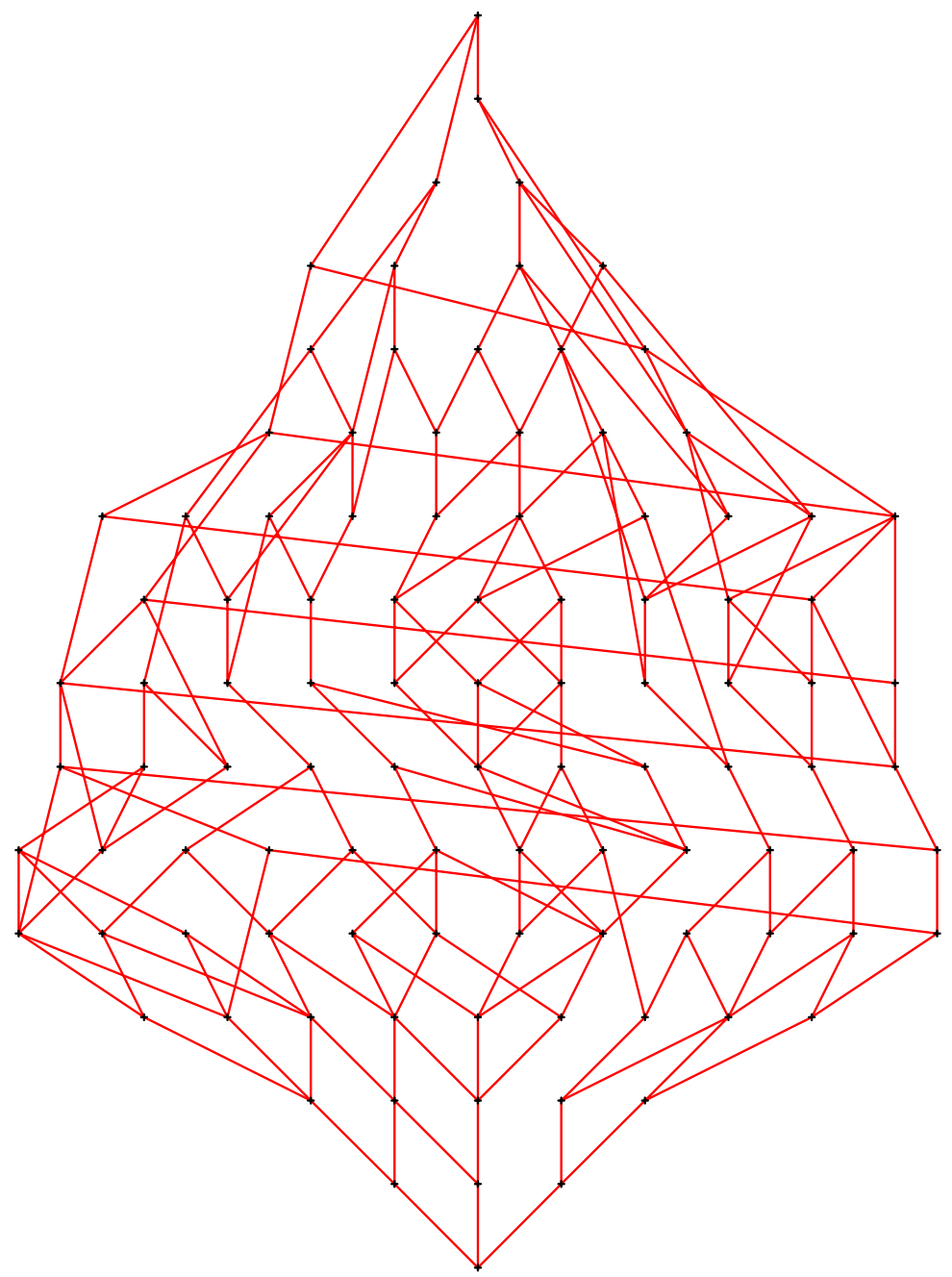

Fig. 11 The Hasse-diagram of $\operatorname{HST}_{1}(10,6)$ generated by an unpublished maple package of the first author and the Stembridge posets package [42].

second higher Stasheff-Tamari order $\operatorname{HST}_{2}(n, d)$. In particular, the Hasse diagram for $\operatorname{HST}_{1}(n, d)$, which is the flip graph $\mathscr{G}_{\text {tri }}(\mathbf{C}(n, d))$, is connected.

Figure 11 shows the Hasse-diagram of $\operatorname{HST}_{1}(10,6)$, a non-trivial case for which boundedness was unknown before. 


\subsection{Diameter}

Since the flip graph $\mathscr{G}_{\text {tri }}(\mathbf{C}(n, d))$ is connected, it makes sense to ask for its diameter, that is, how many flips are required to reach a triangulation from any other, in the worst case. We explain here how the following structural result on $\operatorname{HST}_{1}(n, d)$ leads to the exact diameter when $d$ is odd, and diameter bounds when $d$ is even.

Theorem 6.6. [31. Thm. 1.1] There is a one-to-one correspondence between equivalence classes of maximal chains in $\operatorname{HST}_{1}(n, d)$ and triangulations of $\mathbf{C}(n, d+1)$. Two chains are equivalent if their covering relations are flips on identical sets of $d+1$-simplices. This correspondence is induced by mapping each flip in a maximal chain in $\mathrm{HST}_{1}(n, d)$ to the corresponding $(d+1)$-simplex in $\mathbf{C}(n, d+1)$.



Fig. 12 The connection between a chain in $\operatorname{HST}_{1}(6,1)$ (represented by characteristic sections) and an element of $\operatorname{HST}_{1}(6,2)$ (figures from [11 Chp. 5]).

When $d$ is odd, so that $\operatorname{HST}_{1}(n, d)$ is both ranked and bounded, this determines the diameter of $\mathscr{G}_{\text {tri }}(\mathbf{C}(n, d))$ exactly, combining the previous result, Proposition 3.9 . and the following well-known fact.

Proposition 6.7. A bounded ranked poset of rank $r$ has Hasse diagram diameter $r$.

Proof. Every element lies in a maximal chain of length $r$, and hence any pair of elements are contained in a closed cyclic path of $2 r$ edges that concatenates two such maximal chains; thus they lie at distance at most $r$. On the other hand, the unique bottom and top elements are at distance at least $r$. 
Corollary 6.8. [31] Cor. 1.2] For odd $d=2 e-1$, the diameter of the flip graph of $\mathbf{C}(n, d)$ is $\left(\begin{array}{c}n-e-1 \\ e\end{array}\right)$.

Since a triangulation of $\mathbf{C}(n, d+1)$ for $d$ even has no more simplices than there are lower facets of $\mathbf{C}(n, d+2)$ and no fewer simplices than there are upper facets of $\mathbf{C}(n, d+2)$, the same argument at least gives these bounds for the diameter.

Corollary 6.9. [31 Cor. 1.2] For even $d=2 e$, the diameter of the flip graph of $\mathbf{C}(n, d)$ is bounded between $\left(\begin{array}{c}n-e-2 \\ e\end{array}\right)$ and $2\left(\begin{array}{c}n-e-2 \\ e\end{array}\right)$.

\subsection{The case $d=2$ : the rotation graph of binary trees}

In the case where $d=2$, the above diameter bounds show that the diameter of $\mathscr{G}_{\text {tri }}(\mathbf{C}(n, 2))$ is between $n-3$ and $2 n-6$. However, this case has been extremely well-studied under the guise of the rotation graph on binary trees, e.g. in the work of Pallo; see the survey by Dehornoy [12] in this volume for references, and for the close connection with Thompson's group. In particular, the above diameter bound is superseded by the following celebrated result of Sleator, Thurston, and Tarjan.

Theorem 6.10. [40. Thm. 2] The diameter of $\mathscr{G}_{\mathrm{tri}}(\mathbf{C}(n, 2))$ is, for sufficiently large values of $n$, exactly $2 n-10$.

The proof that the diameter is at least $2 n-10$ for sufficiently large $n$ employs the three-dimensional interpretation of flips sketched above: flipping can be seen as shifting a surface from the lower facets of a (not necessarily straight-line) tetrahedron through the tetrahedron all the way to the upper facets of the tetrahedron.

Moreover, a sequence of flips can be seen as moving a surface all the way through a three-dimensional triangulation, consisting of one tetrahedron per flip and having one triangulation as the bottom and the other triangulation as the top surface. If one could show that there are triangulations of an $n$-gon so that the three-dimensional space between them needs at least $2 n-10$ tetrahedra to be triangulated, then the claim would follow. And indeed: by embedding the situation in hyperbolic geometry (where volumes of simplices are bounded!), Sleator, Tarjan, and Thurston established the lower bound along these lines. Along their way, they had to master a wealth of technical difficulties, though. No combinatorial or more intuitive proof has been given of this lower bound to date.
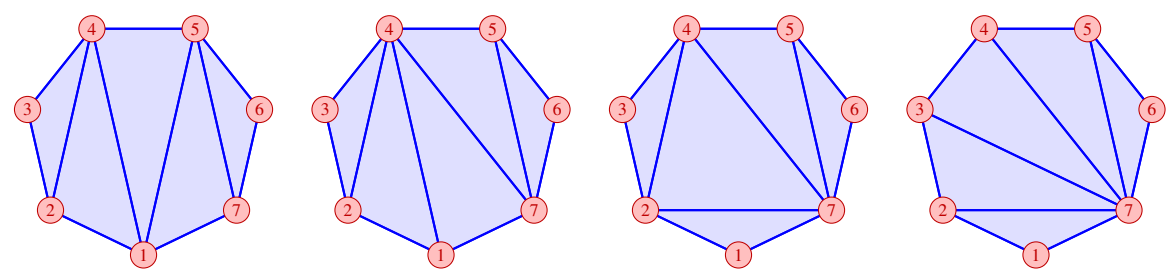

Fig. 13 Flipping (from left to right) to the standard triangulation with respect to vertex 7. 
On the other hand, their argument for the diameter upper bound of $2 n-10$ is easy enough to reproduce here. Pick an arbitrary vertex $\mathbf{p}$ of an $n$-gon with $n>12$ and an arbitrary triangulation $\mathscr{T}$. Unless $\mathbf{p}$ lies in all possible interior edges, that is, its degree $\operatorname{deg}_{\mathscr{T}}(\mathbf{p})$ in the interior edge graph of $\mathscr{T}$ is $n-3$, we can find a flip that increases the degree of $\mathbf{p}$ by one. (In that case, not all adjacent triangles in the star of $\mathbf{p}$ in $\mathscr{T}$ can form a non-convex quadrilateral.) Thus, we need at most $n-3-\operatorname{deg}_{\mathscr{T}}(\mathbf{p})$ flips to transform $\mathscr{T}$ into the unique triangulation with $\operatorname{deg}_{\mathscr{T}}(\mathbf{p})=n-3$, the standard triangulation with respect to $\mathbf{p}$. The same holds for any other triangulation $\mathscr{T}^{\prime}$, so that the flip distance $\operatorname{dist}\left(\mathscr{T}, \mathscr{T}^{\prime}\right)$ between $\mathscr{T}$ and $\mathscr{T}^{\prime}$ is at most

$$
\operatorname{dist}\left(\mathscr{T}, \mathscr{T}^{\prime}\right) \leq \min _{\mathbf{p}} 2 n-6-\operatorname{deg}_{\mathscr{T}}(\mathbf{p})-\operatorname{deg}_{\mathscr{T}^{\prime}}(\mathbf{p})
$$

If one uses the worst case of this relation as an upper bound, one can not get past $2 n-6$. However, symmetry comes to our aid: Since every triangulation of an $n$-gon has $n-3$ interior edges, the average interior-edge degree of a vertex is $(2 n-6) / n=2-6 / n$. Summarized:

$$
\operatorname{dist}\left(\mathscr{T}, \mathscr{T}^{\prime}\right) \leq 2 n-6-2+6 / n-2+6 / n=2 n-10+12 / n .
$$

Since $n>12$ and the distance is integral, the claim follows.

\section{Subdivisions and the Baues problem}

We have already seen, in the discussion of Möbius functions for $\operatorname{HST}_{2}(n, d)$ in Section 5 , the relevance of polytopal subdivisions $\mathscr{S}$ of $\mathbf{C}(n, d)$ which are coarser than triangulations, and the importance of the refinement ordering on them.

The flip graph $\mathscr{G}_{\text {tri }}(\mathbf{A})$ is a one-dimensional object built from these triangulations and bistellar flips relating them. It turns out that bistellar flips can also be thought of as subdivisions which are only slightly coarser than triangulations, namely those that have exactly two refinements, both triangulations. They form part of a larger structure, the Baues poset, built from all subdivisions. The connectivity question for $\mathscr{G}_{\text {tri }}(\mathbf{A})$ is closely related to the question of homotopy type for this Baues poset. We discuss this somewhat informally here - see [36] for further discussion and references.

\subsection{Subdvisions and secondary polytopes}

Polytopal subdivisions of the convex hull of a point configuration A, using only vertices in A, already appeared naturally in the work of Gelfand, Kapranov, and Zelevinsky [19, 20] on the secondary polytope of $\mathbf{A}$ that was discussed in Section 6.2. the face poset of the second polytope is exactly the poset of all regular polytopal subdivisions $\mathscr{S}$ of the convex hull of $\mathbf{A}$, ordered by refinement. See Figure 14 for 
the example of a pentagon (isomorphic to $\mathbf{C}(5,2)$ ). See also [11, Chp. 5] for a more elementary introduction into this theory.
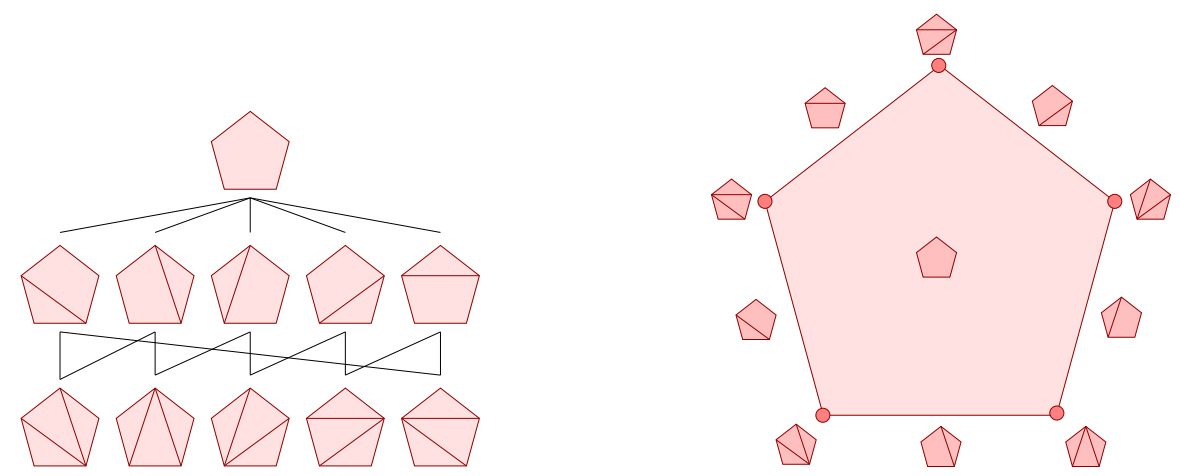

Fig. 14 The refinement poset of a five-gon is isomorphic to the face lattice of its secondary polytope (in this case also a five-gon); figures from [11, Chp. 5].

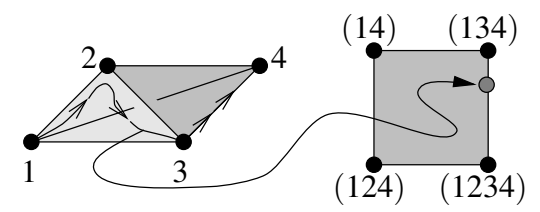

Fig. 15 A path in a tetrahedron and the corresponding cell in the square (figure from [29]).

\subsection{Baues's original problem}

Meanwhile, a conjecture of Baues in the model theory of loop spaces [5] motivated Billera, Kapranov, and Sturmfels [6] to generalize this subdivision poset. We give here a rough idea of Baues's goal, before explaining their generalization.

The loop space $\Omega X$ of a base-pointed topological space $(X, x)$ has elements which are closed paths $\gamma$ in $X$ starting and ending at $x$, equipped with a certain topology. If $X$ happens to come from a simplicial complex, that is, it is glued from simplices, then one might hope to model $\Omega X$ via some type of cell complex; this idea goes back to J. F. Adams [1] who applied it to compute the homology of $\Omega X$.

To this end, consider a piece of a closed path $\gamma$ inside a $d$-simplex, with vertices numbered $\{0,1,2, \ldots, d\}$, with $\gamma$ entering each visited (open) face at its minimal vertex and exiting at its maximal vertex $d$. Moreover, we require that it enters the simplex at vertex 0 and exits at vertex $d$. The various substantially distinct options 
for how this piece of $\gamma$ can traverse the simplex (in terms of visited open faces) can be modeled by a $(d-1)$-cube: the extreme possibilities are edge paths with increasing vertex labels in the simplex, which biject with vertices of a cube: the vertices 1 through $d-1$ of the simplex that are visited by $\gamma$ determine the ones in the coordinates of the vertex of the cube. All intermediate options where $\gamma$ can wander specify in a rather obvious way faces of the cube, where a path meeting the interior of the simplex corresponds to the improper face of the cube, that is, the whole cube.

Thus, one might think that the loop space of a simplicial complex can be modeled by a cubical complex. As always, there are technical subtleties, one of which is that a certain structure must have the homotopy type of a sphere for things to work. Baues conjectured that this structure actually always does have the homotopy type of a sphere.
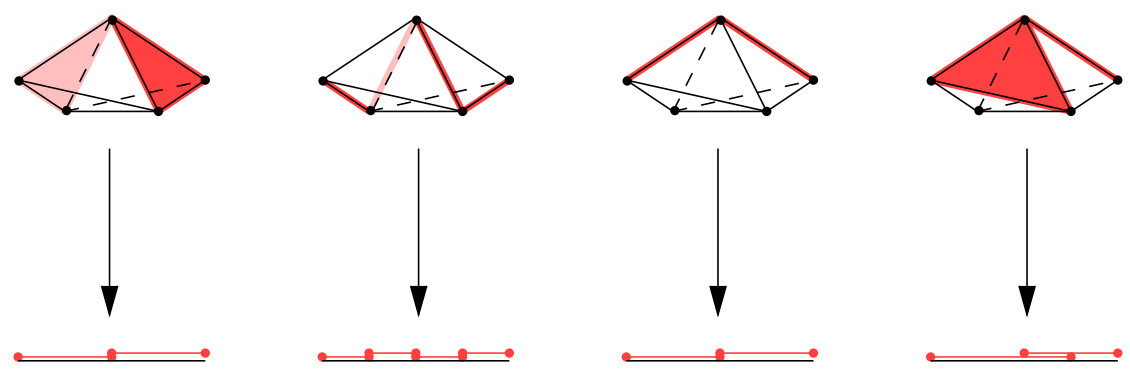

Fig. 16 How cellular strings in the bipyramid project to compatible subdivisions of the line; the rightmost set of faces is not a cellular string, because the projections of those faces overlap (figure derived from a figure in [29 Chap. 1]).

\subsection{Cellular strings and the generalized Baues problem}

Billera, Kapranov, and Sturmfels [6] discovered that the structure Baues was after is an example of the following construction.

Definition 7.1. Consider a $d^{\prime}$-dimensional polytope $P$ and linear functional $\mathbb{R}^{d^{\prime}} \stackrel{\pi}{\rightarrow} \mathbb{R}^{1}$ taking distinct values $\pi(v) \neq \pi\left(v^{\prime}\right)$ whenever $v, v^{\prime}$ are vertices lying on an edge of $P$.

Say that a subdivision of the line segment $\pi(P)$ in $\mathbb{R}^{1}$ into consecutive intervals $\left[v_{0}, v_{1}\right],\left[v_{1}, v_{2}\right], \ldots,\left[v_{\ell-1}, v_{\ell}\right]$ is $\pi$-compatible $\rfloor^{3}$ if, for each $i=1,2, \ldots, \ell$, one can

\footnotetext{
${ }^{3}$ The original term " $\pi$-induced" in [7, 6] was modified in [11] to " $\pi$-compatible" because, in general, there are many subdivisions that are projections of faces under $\pi$, induced by the corresponding cellular strings and $\pi$, not $\pi$ alone.
} 
assign a face $F_{i}$ of $P$ for which $\pi\left(F_{i}\right)=\left[v_{i}, v_{i+1}\right]$. In fact, identify the subdivision with the sequence of faces $\left(F_{1}, \ldots, F_{\ell}\right)$ in $P$. Call this sequence a $\pi$-cellular string in $P$.

For example, among the $\pi$-cellular strings one finds all $\pi$-monotone edge paths from the $\pi$-minimizing vertex to the $\pi$-maximizing vertex of $P$, but one also has $\pi$-cellular strings that take steps through faces which are higher-dimensional than edges; see Figure 16

One defines a refinement ordering on all such $\pi$-cellular strings in $P$ via containment of faces, which gives a poset that was baptized the Baues poset of $P$ and $\pi$. The result that triggered a whole line of research was this.

Theorem 7.2. [6]. For any $d^{\prime}$-dimensional polytope and linear functional $\mathbb{R}^{d^{\prime}} \stackrel{\pi}{\rightarrow} \mathbb{R}^{1}$ as above, the Baues poset is homotopy equivalent to a sphere of dimension $d^{\prime}-2$.

Billera, Kapranov, and Sturmfels also defined a Baues poset of $\pi$-compatible subdivisions for any linear projection $\pi$ of a $d^{\prime}$-dimensional polytope $P$ to a $d$ dimensional polytope $\pi(P)$ for some $d<d^{\prime}$. The following question arose naturally.

Question 7.3 (Generalized Baues Problem (GBP)). For a $d^{\prime}$-polytope $P$ and for any linear projection $\pi$ to $\mathbb{R}^{d}$, does the (Generalized) Baues poset of $P$ and $\pi$ have the homotopy type of a $d^{\prime}-d-1$-sphere?

At the time when this question was phrased it had almost the status of a conjecture. This thinking was fueled by the work of Billera and Sturmfels on the theory of fiber polytopes [7], generalizing Gelfand, Kapranov and Zelevinsky's secondary polytopes. The fiber polytope of the projection $\pi$ out of $P$ distinguishes geometrically a certain subposet of the $\pi$-compatible subdivisions $\mathscr{S}$ of the image polytope $\pi(P)=: Q$, namely those subdivisions which are $\pi$-coherent: one requires that the collection of faces $\left\{F_{i}\right\}$ of $P$ projecting to the subdivision $\mathscr{S}$ does not "wrap around $P$ ", in the sense that there exists a single linear functional $g$ on the $\left(d^{\prime}-d\right)$-dimensional real space $\operatorname{ker}(\pi)$ so that the union $\cup_{i} F_{i}$ is exactly the union over all the points $q$ in $\pi(P)$ of the $g$-maximizing subfaces of the $\left(d^{\prime}-d\right)$-dimensional polytopal fibers $\pi^{-1}(q) \cap P$.

Denoting the subposet of $\pi$-coherent subdivisions the coherent (generalized) Baues poset of $P$ and $\pi$, one has the following striking result.

Theorem 7.4. [7] The coherent generalized Baues poset of $P$ and $\pi$ is always isomorphic to the face lattice of a polytope, the fiber polytope of $P$ and $\pi$. In particular, this subposet is homeomorphic to a $d^{\prime}-d-1$-sphere.

In the example of Figure 17 we see that the poset of coherent compatible subdivisions (solid covering relations) is indeed a proper sub-poset of the Baues poset; this sub-poset is isomorphic to a hexagon whereas the whole poset is only homotopy equivalent to a 1-sphere. By Theorem 7.4, the subdivisions connected by dashed covering relations cannot be compatible, because they lie only in chains that are too long to appear in the face lattice of a 2-dimensional polytope. Note that though the bipyramid is isomorphic to $\mathbf{C}(5,3)$, the indicated projection is not the canonical projection between cyclic polytopes, since the induced order of vertices is $2,1,3,5,4$, as opposed to $1,2,3,4,5$ in the canonical projection. 


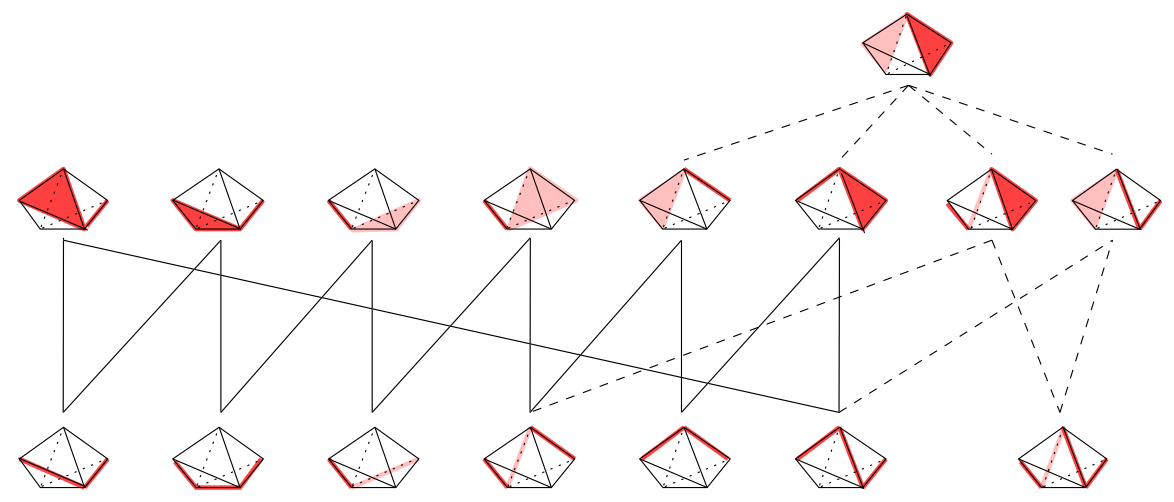

Fig. 17 The (proper part of the) Baues poset of coherent and incoherent cellular strings on a bipyramid, projecting down to compatible subdivisions of a line (example and figure from 47 Chp. 9]; figure also in [29. Chp. 1]).

As time went by with no affirmative answer to Question 7.3 . hope diminished, and finally a surprisingly small counter-example was constructed by Rambau and Ziegler [34].

Theorem 7.5. [34 Thm. 1.5] There is a generic projection $\pi$ of a 5-polytope $P$ with 10 vertices to the plane, having a disconnected generalized Baues poset. In particular, its generalized Baues poset is not homotopy equivalent to a 2-sphere.

This yields counter-examples for any set of larger parameters $d, d^{\prime}$ with $d>2$ and $d^{\prime}-d>2$ by standard constructions. For the only missing parameters $d^{\prime}-d \leq 2$, an affirmative answer could be given, with an involved proof, though:

Theorem 7.6. [34 Thm. 1.4] For $d^{\prime}=d+2$, the generalized Baues poset always has the homotopy type of a 1-sphere.

Thus one has a recurring dichotomy: geometrically distinguished subdivisions form friendly structures, whereas the general subdivisions do not.

On the other hand, as in the case of triangulations, there is a family of particularly friendly polytopes where everything is nice, and it is again - the cyclic polytopes. Work of several authors showe ${ }^{4}$ that the canonical projections between cyclic polytopes have indeed well-behaved generalized Baues posets.

Theorem 7.7. [33] 3] For all $d^{\prime}>d$, the generalized Baues poset of the canonical projection from $\mathbf{C}\left(n, d^{\prime}\right)$ to $\mathbf{C}(n, d)$ has the homotopy type of a $d^{\prime}-d-1$-sphere.

\footnotetext{
${ }^{4}$ For some of the history on the progress toward this result, see [36 $\left.\$ 4\right]$.
} 


\section{Connection to the higher Bruhat orders}

We discuss here the higher Bruhat orders $B(n, k)$ of Manin and Schechtman [25] generalizing the weak Bruhat order $B(n, 1)$ on the set $\mathfrak{S}_{n}$ of all permutations of $n$ letters. Their intimate connection to the higher Stasheff-Tamari orders appears already in the original paper of Kapranov and Voevodsky, who discuss [24, \$4] a poset map $B(n, k) \rightarrow \operatorname{HST}_{1}(n+2, k+1)$ generalizing the classical poset surjection from the weak Bruhat order on $\mathfrak{S}_{n}$ to the Tamari order on triangulations of $\mathbf{C}(n+2,2)$; see the survey by Reading [35] and by Hohlweg [21] for more perspectives and different generalizations of this map. Further discussion of higher Bruhat orders, higher Stasheff-Tamari orders, and the poset map between them appears in [36, §6].

\subsection{Definition of higher Bruhat orders}

One can think of the higher Bruhat orders $B(n, k)$ as orders on orders on orders $\ldots$ of subsets. When defined for general $k$, they can seem a bit technical. Here we choose instead to work our way up from $k=0,1,2, \ldots$

Example 8.1. When $k=0$, the (lower!) Bruhat order $B(n, 0)$ is the Boolean algebra $2^{\{1,2, \ldots, n\}}$. Thus it is isomorphic to the two lower Stasheff-Tamari orders on the set of triangulations of of $\mathbf{C}(n+2,1)$, as described in Example 3.4

Note that this isomorphism is most natural if one renumbers the vertices on the line segment $\mathbf{C}(n+2,1)$ as $0,1,2, \ldots, n+1$, rather than our usual numbering $1,2, \ldots, n+2$, so that the internal vertices are labelled $\{1,2, \ldots, n\}$.

Also, note that one can think of $B(n, 0)=2^{\{1,2, \ldots\}}$ in two ways:

- It is the transitive closure of the relation $S<T$ whenever $S \subset T$ and $|T|=|S|+1$. This is analogous to $\operatorname{HST}_{1}(n+2,1)$.

- One has $S \leq T$ for two subsets $S, T$ in $2^{\{1,2, \ldots, n\}}$ if $S \subseteq T$. This is analogous to $\operatorname{HST}_{2}(n+2,1)$. When we wish to emphasize this analogy, we will borrow Ziegler's notation from [46] where he denotes this inclusion order $B_{\subseteq}(n, 0)$, to distinguish it from the definition via single-step inclusion.

Example 8.2. When $k=1$, the poset $B(n, 1)$ is actually the weak Bruhat order on the symmetric group $\mathfrak{S}_{n}$. As a set, it consists of all maximal chains

$$
\varnothing \subset\left\{w_{1}\right\} \subset\left\{w_{1}, w_{2}\right\} \subset \cdots \subset\left\{w_{1}, w_{2}, \ldots, w_{n-1}, w_{n}\right\}=\{1,2, \ldots, n\}
$$

of elements in $B(n, 0)$. Such chains biject with the linear orders $w=\left(w_{1}, \ldots, w_{n}\right)$ in which the elements are added, which can be read as permutations $w$ in $\mathfrak{S}_{n}$.

To order $B(n, 1)$, recall the (left-)inversion set $\operatorname{Inv}_{2}(w)$ of $w$ is the collection of pairs $i<j$ (which we will call 2-packets) for which $j$ appears before $i$ in the order $w$. Define $w \leq w^{\prime}$ in $B \subseteq(n, 1)$ if $\operatorname{Inv}_{2}(w) \subseteq \operatorname{Inv}_{2}(w)$. On the other hand, one can define the single-step ordering $B(n, 1)$ as the transitive closure of the relation $w<w^{\prime}$ if 
$\operatorname{Inv}_{2}(w) \subset \operatorname{Inv}_{2}\left(w^{\prime}\right)$ and $\left|\operatorname{Inv}_{2}\left(w^{\prime}\right)\right|=\left|\operatorname{Inv}_{2}(w)\right|+1$. It is a classical result that these two orders on $\mathfrak{S}_{n}$ are the same, and define the weak Bruhat order.


note that inversion sets $\operatorname{Inv}_{2}(w)$ of permutations $w$ are not arbitrary subsets of all 2-packets $(\{1,2, \ldots, n\})$ : given any 3-packet $i<j<k$, transitivity forces that if $i j, j k$ lie in $\operatorname{Inv}_{2}(w)$ or if both are absent from $\operatorname{Inv}_{2}(w)$, then the same must be true for $i k$. Said differently, for each 3-packet $i<j<k$, an inversion set $\operatorname{Inv}_{2}(w)$ must intersect the lexicographic order on the 2 -packets

$$
i j, \quad i k, \quad j k
$$

in either an initial segment, or a final segment.

Example 8.3. Things become more interesting when $k=2$. Again $B(n, 2)$ can be derived from consideration of maximal chains $c$ in $B(n, 1)\left(=B_{\subseteq}(n, 2)\right)$. Such chains are sequences of permutations

$$
e=w^{(0)}<w^{(1)}<\cdots<w^{\left(\left(\begin{array}{l}
n \\
2
\end{array}\right)\right)}=w_{0}
$$

from the identity $e$ to the longest element $w_{0}=(n, n-1, \ldots, 2,1)$, in which one adds one element to $\operatorname{Inv}_{2}\left(w^{(i)}\right)$ at each stage. These maximal chains correspond to reduced decompositions for $w_{0}$ in terms of the adjacent transposition Coxeter generators $s_{i}=(i, i+1)$ for $\mathfrak{S}_{n}$.

One can derive from such a chain $c$ a 3-packet inversion $\operatorname{set} \operatorname{Inv}_{3}(c)$ by considering for each 3-packet $i<j<k$ the two possible orders in which its 2-packet subsets $i j, i k, j k$ are added to the sets $\operatorname{Inv}_{2}\left(w^{(i)}\right)$ for $i=1,2, \ldots,\left(\begin{array}{c}n \\ 2\end{array}\right)$ : either they are added in the lexicographic order from (6) and one decrees $i j k \notin \operatorname{Inv}_{3}(c)$, or they are added in the reverse of this order and one decrees $i j k \in \operatorname{Inv}_{3}(c)$.

As a set $B(n, 2)$ is defined to be all equivalence classes $\bar{c}$ of such chains $c$, where $c, c^{\prime}$ are equivalent if $\operatorname{Inv}_{3}(c)=\operatorname{Inv}_{3}\left(c^{\prime}\right)$. In terms of reduced words for $w_{0}$, this is the same as equivalence under commuting braid moves $s_{i} s_{j}=s_{j} s_{i}$ for $|i-j| \geq 2$.

Similarly to $B_{\subseteq}(n, 1)$, define $\bar{c} \leq \bar{c}^{\prime}$ in $B_{\subseteq}(n, 2)$ if $\operatorname{Inv}_{3}(\bar{c}) \subseteq \operatorname{Inv}_{3}\left(\bar{c}^{\prime}\right)$. On the other hand, one can define the single-step ordering $B(n, 2)$ as the transitive closure of the covering relation $\bar{c} \lessdot \bar{c}^{\prime}$ if their 3-packet inversion sets are nested and differ in cardinality by one.

It is no longer obvious that these two orders $B(n, 2)$ and $B_{\subseteq}(n, 2)$ on the set are the same; this nontrivial fact was proven by Felsner and Weil [18].

And it is again true that inversions sets $\operatorname{Inv}_{3}(\bar{c})$ are not arbitrary subsets of all 3 -packets $\left(\begin{array}{rl}\{1,2, \ldots, n\} \\ 3\end{array}\right)$ : given any 4-packet $i_{1}<i_{2}<i_{3}<i_{4}$, they are characterized by a nontrivial biconvexity condition [46, Lemma 2.4] asserting that $\operatorname{Inv}_{3}(\bar{c})$ must intersect the lexicographic order on the 3-packets within this 4-packet

$$
i_{1} i_{2} i_{3}, \quad i_{1} i_{2} i_{4}, \quad i_{1} i_{3} i_{4}, \quad i_{2} i_{3} i_{4}
$$

in either an initial segment or a final segment. 
This picture continues, allowing one to derive $B(n, k)$ from considering maximal chains $c$ of elements in $B_{s s}(n, k-1)$. Each such maximal chain can be considered as the sequence of $k$-packets added to the $k$-inversion sets of the elements in the chain. For each fixed $(k+1)$-packet $S$, its subset $k$-packets are added either in lexicographic, or reverse lexicographic order, as one proceeds up the chain, and one uses this to decree whether the $(k+1)$-packet does not or does lie in $\operatorname{Inv}_{k+1}(c)$.

One defines $B(n, k)$ as a set to be the equivalence classes $\bar{c}$ of these chains $c$ in $B_{s s}(n, k-1)$ having the same $(k+1)$-packet inversion sets $\operatorname{Inv}_{k+1}(c)$. One orders the set $B(n, k)$ either as $B \subseteq(n, k)$ via inclusion of the sets $\operatorname{Inv}_{k+1}(c)$, or denotes the analogous poset $B(n, k)$ defined via single-step inclusion.

Although these definitions are recursive, one can also make them nonrecursive; see Ziegler [46]. On the other hand, the recursive description builds in a classical result by Manin and Schechtman on how the structures of the higher Bruhat orders $B(n, k)$ are intertwined for different $k$; Theorem 6.6 was actually inspired by the following older result.

Theorem 8.4. [25. Thm. 3] There is a one-to-one correspondence between certain equivalence classes of maximal chains in $B(n, k)$ and elements of $B(n, k+1)$.

On the other hand, when $k \geq 3$, a subtlety appears, in that Ziegler shows [46, Theorem 4.5] that $B_{s s}(n, 3)$ and $B_{\subset}(n, 3)$ do not coincide for $n \geq 8$. In light of this fact, and the existence of the map $B(n, k) \rightarrow \operatorname{HST}_{1}(n+2, k+1)$, to be discussed in Section 8.3 below, the resolution of Problem 3.3 becomes even more interesting.

\subsection{Some geometry of higher Bruhat orders}

The geometry that originally motivated Manin and Schechtman to define $B(n, d)$ comes from generalizations of the classical braid arrangement known as discriminantal arrangements. We will not discuss this here, but instead focus on the interpretation of $B(n, d)$ via tilings of the $d$-dimensional cyclic zonotope $\mathbf{Z}(n, d)$, defined to be the Minkowski sum

$$
\mathbf{Z}(n, d)=\left\{\sum_{i=1}^{n} c_{i} v_{d}\left(t_{i}\right):-1 \leq c_{i} \leq+1\right\}
$$

consisting of all $[-1,+1]$ combinations of $n$ distinct vectors $v_{d}\left(t_{1}\right), \ldots, v_{d}\left(t_{n}\right)$ lying on the moment curve in $\mathbb{R}^{k}$, with $t_{1}<\cdots<t_{n}$, as usual.

This interpretation is essentially stated without proof in the paper of Kapranov and Voedvodsky [24, Theorem 4.9]. It was carefully justified and explained later by Thomas in [44. Thm. 2.1], as well as by Ziegler's discussion following [46, Theorem 4.1] when one takes into account the equivalence between zonotopal tilings and oriented matroid single-element liftings given by the Bohne-Dress Theorem [9, Theorem 2.2.13].

The story begins with the observation that any zonotope which is a Minkowski sum $\sum_{i=1}^{n}[-1,+1] v_{i}$ generated by $n$ vectors $v_{1}, \ldots, v_{n}$ in $\mathbb{R}^{d}$ is simply the projection 


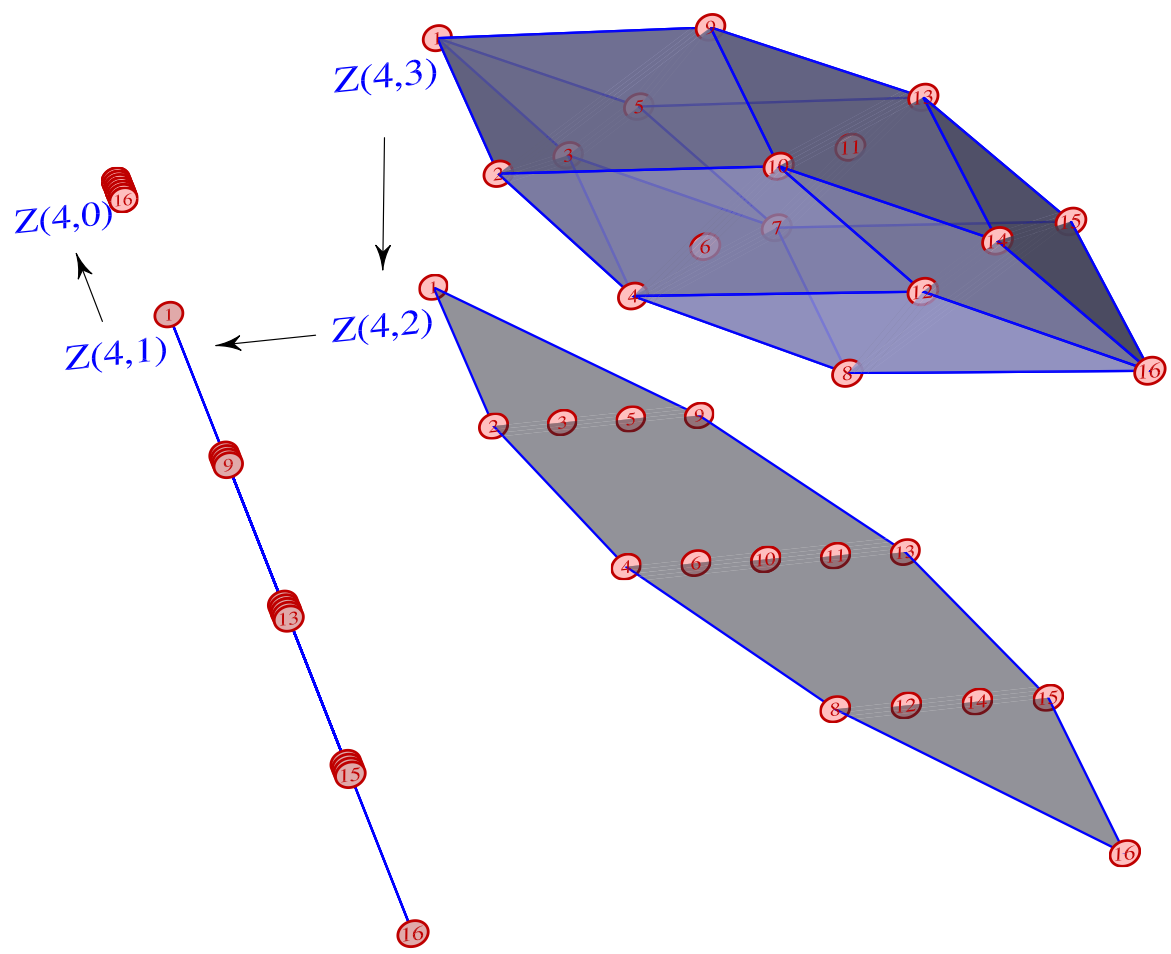

Fig. 18 Cyclic zonotopes $\mathbf{Z}(4,3), \mathbf{Z}(4,2), \mathbf{Z}(4,1)$, and $\mathbf{Z}(4,0)\left(2^{4}=16\right.$ repeated points at the origin) together with the canonical projections forgetting the last coordinate. Note that already $\mathbf{Z}(4,3)$ has interior points, namely 6 and 11, and already $\mathbf{Z}(4,2)$ has repeated points, namely 7 and $10 . \mathbf{Z}(4,4)$ is simply a 4 -cube.

of the $n$-cube $[+1,-1]^{n}$ in $\mathbb{R}^{n}$ generated by the standard basis vectors $e_{1}, \ldots, e_{n}$, under the linear projection $\mathbb{R}^{n} \rightarrow \mathbb{R}^{d}$ that sends $e_{i}$ to $v_{i}$. Because of this, one has a natural tower of projections $\mathbf{Z}\left(n, d^{\prime}\right) \rightarrow \mathbf{Z}(n, d)$, depicted for $n=4$ in Figure 18 , analogous to the tower of projections $\mathbf{C}\left(n, d^{\prime}\right) \rightarrow \mathbf{C}(n, d)$ discussed in Section 2 and depicted in Figure 1 .

To explain the interpretation of $B(n, d)$ in terms of tight zonotopal tilings of $\mathbf{Z}(n, d)$, that is, tilings by subzonotopes which cannot be further refined, we will work our way up from the low-dimensional cases, where the geometry is simpler. For a careful discussion of the definitions of zonotopal tilings, we refer the reader to Richter-Gebert and Ziegler [37], Billera and Sturmfels [7, §4], or DeLoera, Rambau and Santos [11, §9.1.2].

Example 8.5. When $d=0$, each vector $v_{0}\left(t_{i}\right)$ lies at the origin which is the unique point in $\mathbb{R}^{0}$, and equals the zero-dimensional zonotope $\mathbf{Z}(n, 0)$. However, we regard the point $\mathbf{Z}(n, 0)$ as having $2^{n}$ different labels by subsets $S$ of $\{1,2, \ldots, n\}$, each corresponding to the vertex of the $n$-cube $Z(n, n)$ that projects to it. Thus, an element 
$S$ of $B(n, 0)=2^{\{1,2, \ldots, n\}}$, is a choice of such a label, and is considered a zonotopal tiling of $\mathbf{Z}(n, 0)$. Alternatively, it gives a section of the map $\mathbf{Z}(n, n) \stackrel{\pi}{\rightarrow} \mathbf{Z}(n, 0)$.

Note also that the covering relation between subsets $S \lessdot S^{\prime}$ in $B(n, 0)$ corresponds to two vertices lying along an edge of the $n$-cube.

Example 8.6. When $d=1$, each vector $v_{1}\left(t_{i}\right)=t_{i}$ points along the $\left(x_{1}\right.$-)axis of $\mathbb{R}^{1}$, and $Z(n, 1)$ is the line segment whose two endpoints $v_{\min }, v_{\max }$ are $\pm\left(t_{1}+\cdots+t_{n}\right)$. A tight zonotopal tiling of $Z(n, 1)$ is a sequence of intervals

$$
\begin{aligned}
& {\left[v_{\min }, v_{\min }+2 t_{w_{1}}\right],} \\
& {\left[v_{\min }+2 t_{w_{1}}, v_{\min }+2 t_{w_{1}}+2 t_{w_{2}}\right],} \\
& \ldots, \\
& {\left[v_{\min }+2 t_{w_{1}}+2 t_{w_{2}}+\cdots+2 t_{w_{n-1}}, v_{\max }\right]}
\end{aligned}
$$

corresponding to a permutation $w=\left(w_{1}, \ldots, w_{n}\right)$ in $\mathfrak{S}_{n}$, or an element of $B(n, 1)$; see Example 8.2.

On the other hand, such permutations or elements of $B(n, 1)$ correspond to maximal chains in $B(n, 0)$, that is, sequences of nested subsets as in (5), and hence by our observation for $d=0$, to edge-paths in the cube $\mathbf{Z}(n, n)$ which proceed in a monotone fashion from the vertex labelled by the empty set $\varnothing$ to the vertex labelled by $\{1,2, \ldots, n\}$. In other words, they give sections of the map $\mathbf{Z}(n, n) \stackrel{\pi}{\rightarrow} \mathbf{Z}(n, 1)$. See Figure 21 and following for some examples of such edges paths with $n=3$.

Note also that covering relation between two permutations $w \lessdot w^{\prime}$ in $B(n, 1)$ corresponds to two monotone edge paths in the cube $\mathbf{Z}(n, n)$ that differ only in two adjacent steps that proceed in opposite ways around a quadrilateral face of the cube

Example 8.7. Again, things become interesting when $d=2$. Now the vectors $v_{2}\left(t_{i}\right)$ in $\mathbb{R}^{2}$ generate a zonotopal polygon $\mathbf{Z}(n, 2)$, that is, a centrally symmetric $2 n$-gon.

An element of $B(n, 2)$ can be thought of as a maximal chain of permutations in $B(n, 1)$ as in 77 , up to a certain equivalence relation. It is possible to model this equivalence relation in at least two ways. One way considers the associated pseudoline arrangement or wiring diagram, as in Figure 19. whose vertical slices record the permutations in the chain as the ordering of the strands. These diagrams are considered only up to the equivalence relation of isotopies in the plane that never allow one strand to slide over the crossing of two other strands.

The other way considers each permutation $w_{i}$ in the chain as a monotone edge path in the cube, and each covering relation $w_{i} \lessdot w_{i+1}$ in the chain as giving a quadrilateral face of the cube on which the two paths take two adjacent steps that disagree. The union of all such quadrilateral faces is a 2-dimensional surface inside the cube $Z(n, n)$, which is a section of the map $\mathbf{Z}(n, n) \rightarrow \mathbf{Z}(n, 2)$.

The concordance between these two models is that the quadrilateral faces in this 2-dimensional surface map under $\pi$ to a tight zonotopal tiling of the $2 n$-gon $\mathbf{Z}(n, 2)$. This tiling can be recovered as the planar dual graph to the graph given by the pseudoline arrangement, considered as having vertices only at the strand crossings; see Figure 19 




Fig. 19 An element of $B(4,2)$ derived from a maximal chain of permutations in $B(4,1)$, the weak Bruhat order on $\mathfrak{S}_{4}$. The chain of permutations (colored from red to cyan) leads to an arrangement of pseudolines, also called a wiring diagram: horizontal slices have the strands ordered as in the permutations in the chain. The planar dual of the pseudoline graph can be drawn as a tight subdivision of the zonotope $\mathbf{Z}(4,2)$, in which the pseudoline strand $i$ for $i=1,2,3,4$ is dual to the edges of the tiles in the parallelism class labelled by $i$. Moreover, the chain of permutations can be recovered in the zonotopal tiling as a sequence of monotone paths (colored from red to cyan) with covering relations coming from "flipping" the paths "upwards" through a quadrilateral.

This picture continues. The work of Thomas [44, Prop. 2.1], Ziegler [46, Theorem 4.1] shows that an element of $B(n, d)$ can be thought of as unions of $d$-dimensional faces inside the cube $\mathbf{Z}(n, n)$, corresponding to the image of a section of the map $\mathbf{Z}(n, n) \stackrel{\pi}{\rightarrow} \mathbf{Z}(n, d)$, projecting to a tight zonotopal subdivision of $\mathbf{Z}(n, d)$.

One can furthermore show that if one instead associates to these tight zonotopal subdivisions $\mathscr{S}$ of $\mathbf{Z}(n, d)$ a section $s \mathscr{S}$ of the map $\mathbf{Z}(n, d+1) \stackrel{\pi}{\rightarrow} \mathbf{Z}(n, d)$, then one has $\mathscr{S} \leq \mathscr{S}^{\prime}$ in the higher Bruhat order $B_{\subseteq}(n, d)$ exactly when $s_{\mathscr{S}}(x)_{d+1} \leq s_{\mathscr{S}^{\prime}}(x)_{d+1}$ for all $x$ in $\mathbf{Z}(n, d)$; see Figure 20 for this picture of $B_{\subseteq}(4,2)$.

Analogously to the situation for cyclic polytopes $\mathbf{C}(n, d)$, these tight zonotopal subdivisions and the edges between them in the Hasse diagram for $B(n, d)$ are special cases of the more general notion of a zonotopal subdivision of $\mathbf{Z}(n, d)$, which is 


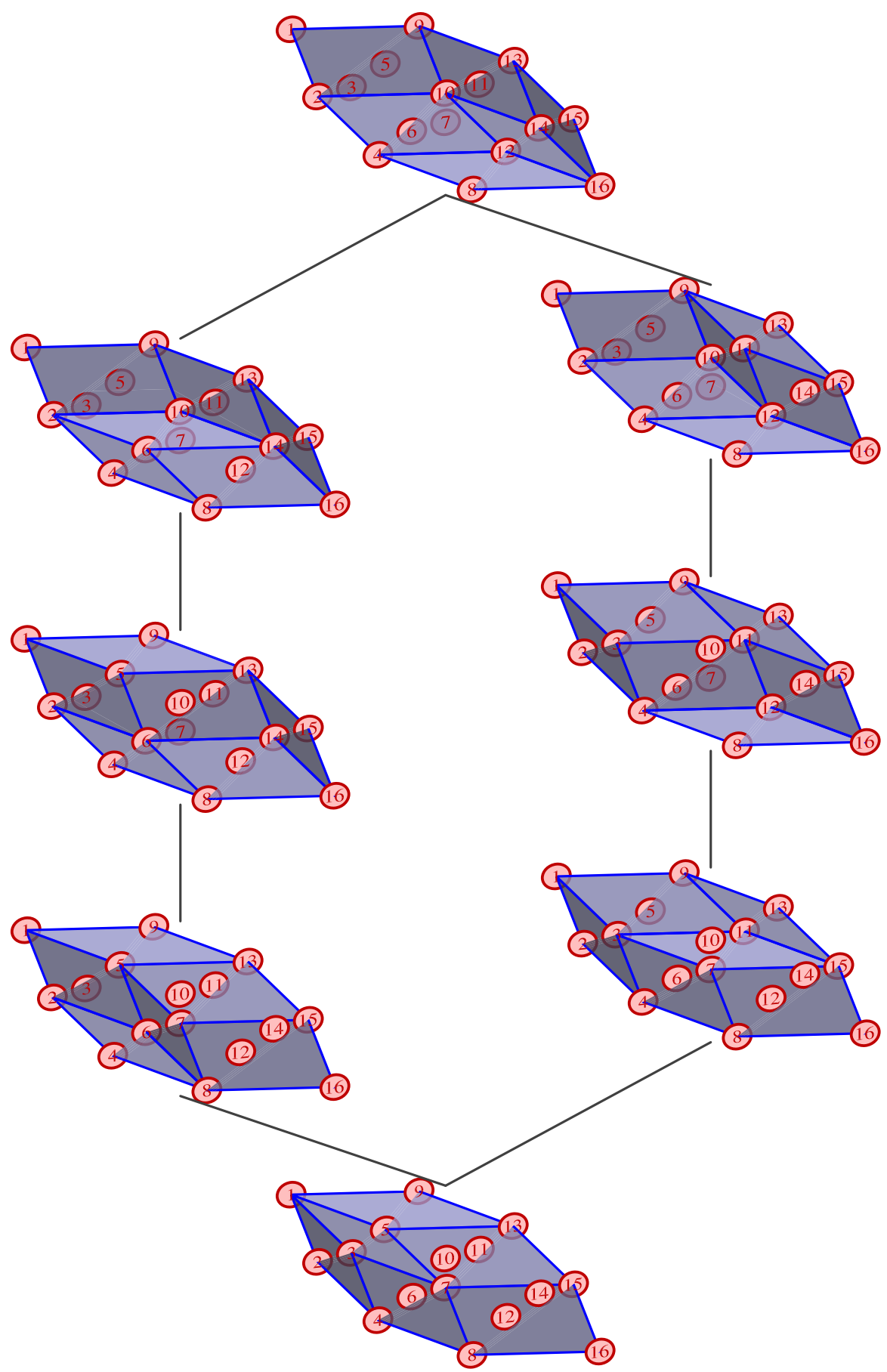

Fig. 20 A picture of $B(4,2)$ with elements drawn as the sections of zonotopal tilings of $\mathbf{Z}(4,2)$ in $\mathbf{Z}(4,3)$, partially ordered by height; it can be seen how the sections, on their way to the top, submerge more and more points. Each chain can be built by stacking cubes, and the cubes corresponding to a chain form a zonotopal tiling of $\mathbf{Z}(4,3)$, which represents an element of $B(4,3)$. 
a $\pi$-compatible subdivision for the projection $\mathbf{Z}(n, n) \stackrel{\pi}{\rightarrow} \mathbf{Z}(n, d)$. There is again a Baues poset of all such subdivisions, ordered by refinement, and the Baues problem asks for its homotopy type. Athanasiadis [2] investigated the Baues problem for all of the canonical projections $\mathbf{Z}\left(n, d^{\prime}\right) \stackrel{\pi}{\rightarrow} \mathbf{Z}(n, d)$, as in Figure 18

Theorem 8.8. [2 Thm. 1.1] For all $d^{\prime}>d$, the generalized Baues poset of the canonical projection from $\mathbf{Z}\left(n, d^{\prime}\right)$ to $\mathbf{Z}(n, d)$ has the homotopy type of a $d^{\prime}-d-1$ sphere.

\subsection{The map from higher Bruhat to higher Stasheff-Tamari orders}

The similarity of the description between the higher Bruhat orders $B(n, k)$ in the last section should make their analogy to the higher Stasheff-Tamari orders $\operatorname{HST}_{1}(n, d)$ apparent.

Tightening the connection, Kapranov and Voevodsky [24] claimed, and later Rambau [31] proved, that there actually is a poset map between them. Later, Thomas shed more light on this connection in [44, §4] (see Figures 21 through 24 for an illustration).

Theorem 8.9. [31 Cor. 8.16]. There is an order-preserving map

$$
B(n, k) \stackrel{f}{\rightarrow} \operatorname{HST}_{1}(n+2, k+1) .
$$

In low dimensions, the map $f$ is familiar. Example 8.1 noted the isomorphisms

$$
B(n, 0)=2^{\{1,2, \ldots, n\}} \cong \operatorname{HST}_{1}(n+2,1) .
$$

In the next dimension up, the map $B(n, 1) \stackrel{f}{\rightarrow} \operatorname{HST}_{1}(n+2,2)$ is the same as the map from the weak Bruhat order on $\mathfrak{S}_{n}$ to the Tamari order on triangulations of $\mathbf{C}(n+2,2)$ discussed in the survey by Reading [35, §1] in this volum 5 ] To describe it in our geometric setting, one must assign a triangulation of $\mathbf{C}(n+2,2)$ to each permutation $w$ in $\mathfrak{S}_{n}$, or to each monotone edge path in the $n$-cube. To this end, think of $C(n+2,2)$ as labeled by $0,1,2, \ldots, n+1$, with $\{0, n+1\}$ its only upper edge. In the order of the permutation $w$, cut off any remaining vertex $i$ of $\mathbf{C}(n+2,2)$ by inserting the diagonal from its left to its right neighbor. Once all vertices $0<i<n+1$ have been cut off, the set of inserted diagonals forms a triangulation. Note that two distinct permutations can map to the same triangulation because $i, j$ that are adjacent in the permutation but not adjacent during the cut-off procedure can be cut off in an arbitrary order. Compare this with the description of this map in the survey by Reading [35, §1], and in particular, compare [35, Figure 3], with Figures 22 through 24 below.

This $f$ extends (modulo technical details) to a map $B(n, d) \stackrel{f}{\rightarrow} \operatorname{HST}_{1}(n+2, d+1)$ via induction on $d$. Elements of $B(n, d)$ are equivalence classes of maximal chains

${ }^{5}$ This map also appears implicitly in the survey by Hohlweg [21], where it is explained how to embed the associahedron in such a way that its normal fan coarsens that of the permutohedron. 


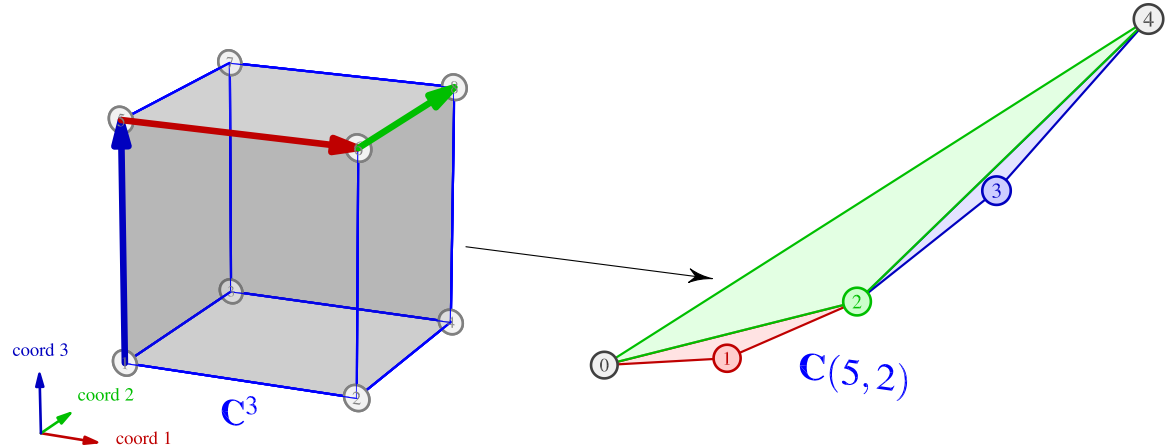

Fig. 21 Each permutation in $B(3,1)$ corresponds to a monotone path in the 3-cube, which induces a triangulation of $\mathbf{C}(5,2)$ by using the order in which the coordinates change as the order in which the vertices 1,2,3 are cut-off by the triangulation. Note that this can be interpreted as an upflip sequence in $\operatorname{HST}_{1}(5,1)$. Thus, what we see here is the flip map $\mathscr{T}_{\text {flip }}$ from [31.

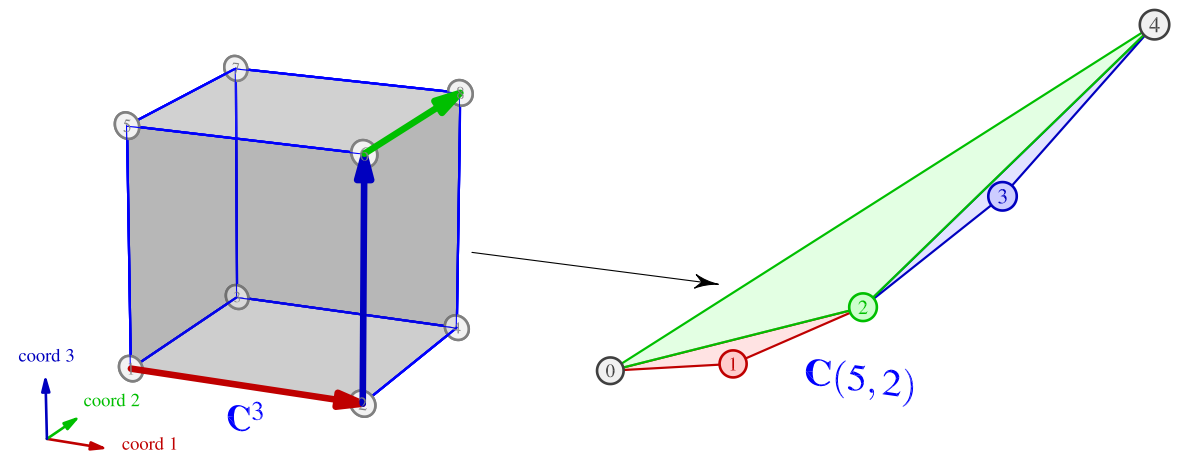

Fig. 22 A different monotone path can lead to an identical triangulation.

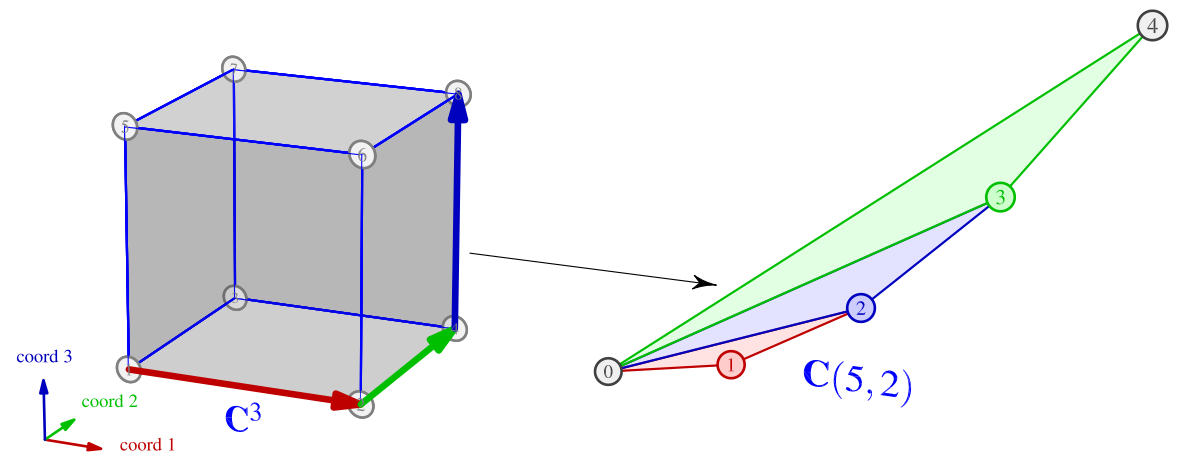

Fig. 23 A different monotone path can also lead to a different triangulation. 

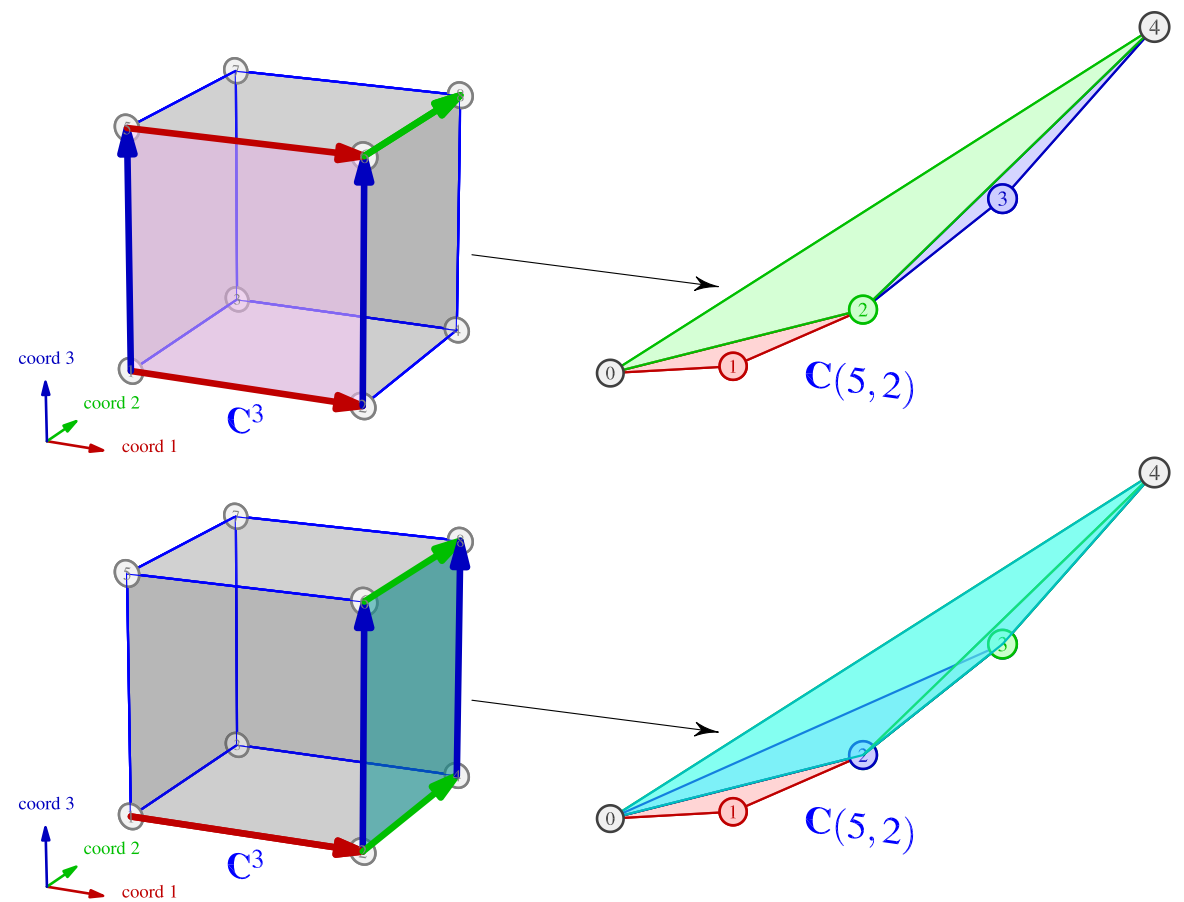

Fig. 24 Monotone paths that differ by a "face flip" (that is, the corresponding permutations are connected by an inversion) lead to triangulations that are either identical or are connected by a bistellar flip.


Fig. 25 Illustration of the inductive structure of the map from higher Bruhat orders to higher Stasheff-Tamari orders: A zonotopal tiling of $\mathbf{Z}(4,2)$ (the one from Figure 19 ) can be traversed upwards by monotone paths (colored from red to cyan), which map to triangulations of $\mathbf{C}(6,2)$ that form a chain (from red to cyan) inducing a triangulation of $\mathbf{C}(6,3)$ consisting of the flip simplices in the chain - determining an element of $\operatorname{HST}_{1}(6,3)$.

$$
c=c_{1} \lessdot c_{2} \lessdot \cdots
$$

of elements $c_{i}$ in $B(n, d-1)$. Each $f\left(c_{i}\right)$ in $\operatorname{HST}_{1}(n+2, d)$ is already defined by induction, and thereby gives a sequence of triangulations of $\mathbf{C}(n+2, d)$ 


$$
f\left(c_{1}\right) \leq f\left(c_{2}\right) \leq \cdots
$$

It can be shown that for each $i$, either $f\left(c_{i}\right)=f\left(c_{i+1}\right)$ or $f\left(c_{i}\right) \lessdot f\left(c_{i+1}\right)$ in the order $\operatorname{HST}_{1}(n+2, d)$. Hence, after eliminating duplicates, the sequence (8) gives a maximal chain in $\operatorname{HST}_{1}(n+2, d)$, and therefore an element of $\operatorname{HST}_{1}(n+2, d+1)$ by Theorem 6.6 This inductive construction is illustrated in Figure 25 .

The results summarized in this section all required technical formal proofs, for which we refrain from presenting any details. However, we close with one problem on the above map $f$, suggested by an assertion from the original paper of Kapranov and Voevodsky [24. Theorem 4.10], but which has so far remained unproven.

Open Problem 8.10. Prove that the map $B(n, d) \stackrel{f}{\rightarrow} \operatorname{HST}_{1}(n+2, d+1)$ is surjective.

\section{Enumeration}

We close with an enumerative question: How large are the posets $\operatorname{HST}_{1}(n, d), \operatorname{HST}_{2}(n, d)$, that is, how many triangulations are there of the cyclic polytope $\mathbf{C}(n, d)$ ?

A few mostly trivial results in this direction are known, such as

- $\mathbf{C}(n, 0), \mathbf{C}(n, 1), \mathbf{C}(n, 2)$ have $n, 2^{n-2}, \frac{1}{n-1}\left(\begin{array}{c}2(n-2) \\ n-2\end{array}\right)$ triangulations, respectively,

- $\mathbf{C}(n, n-1), \mathbf{C}(n, n-2), \mathbf{C}(n, n-3)$, have $1,2, n$ triangulations, respectively.

The following nontrivial result was proven by Azaola and Santos [4].

Theorem 9.1. [4] The number of triangulations of $\mathbf{C}(n, n-4)$ is

$$
\begin{cases}(n+4) \cdot 2^{\frac{n-4}{2}}-n & \text { for } n \text { even, and } \\ \frac{3 n+11}{2} \cdot 2^{\frac{n-5}{2}}-n & \text { for } n \text { odd } .\end{cases}
$$

Another interesting unsolved problem is the following.

Open Problem 9.2. Count the triangulations of $\mathbf{C}(n, 3)$.

How about computer-based enumeration? Table 1 below compiles a few results achieved by the general purpose enumeration program for triangulations TOPCOM [32]. With special purpose codes it should be possible to generate more numbers that can be used to check conjectural enumeration formulas.

\section{References}

1. J. F. Adams, "On the cobar construction", Proceedings of the National Academy of Science $\mathbf{4 2}$ (1956) 409-412.

2. C. Athanasiadis, "Zonotopal subdivisions of cyclic zonotopes", Geometriae Dedicata 86 (2001) 37-57. 


\begin{tabular}{|c|c|c|c|c|c|c|c|c|c|c|c|c|c|c|}
\hline$c \backslash^{d:}$ & & 1 & 2 & 3 & 4 & 5 & 6 & 7 & 8 & 9 & 10 & 11 & 12 & 13 \\
\hline 1 & 1 & 1 & 1 & 1 & 1 & 1 & 1 & 1 & 1 & 1 & 1 & 1 & 1 & 1 \\
\hline 2 & 2 & 2 & 2 & 2 & 2 & 2 & 2 & 2 & 2 & 2 & 2 & 2 & 2 & 2 \\
\hline 3 & 3 & 4 & 5 & 6 & 7 & 8 & 9 & 10 & 11 & 12 & 13 & 14 & 15 & 16 \\
\hline 4 & 4 & 8 & 14 & 25 & 40 & 67 & 102 & 165 & 244 & 387 & 562 & 881 & 1264 & 1967 \\
\hline 5 & 5 & 16 & 42 & 138 & 357 & 1233 & 3278 & 12589 & 35789 & 159613 & 499900 & 2677865 & 9421400 & 62226044 \\
\hline 6 & 6 & 32 & 132 & 972 & 4824 & 51676 & 340560 & 6429428 & & & & & & \\
\hline 7 & 7 & 64 & 429 & 8477 & 96426 & 5049932 & 132943239 & & & & & & & \\
\hline 8 & 8 & 128 & 1430 & 89405 & 2800212 & & & & & & & & & \\
\hline 9 & 9 & 256 & 4862 & 1119280 & 116447760 & & & & & & & & & \\
\hline 10 & & 516 & 16796 & 16384508 & & & & & & & & & & \\
\hline 11 & & 1028 & 58786 & 276961252 & & & & & & & & & & \\
\hline
\end{tabular}

Table 1 Some computations done with TOPCOM [32] for some dimensions $d$ and some codimensions $c:=n-d$; the computation of the largest numbers in the table for $\mathbf{C}(13,6)$ and $\mathbf{C}(14,3)$ needed around $40 \mathrm{~GB}$ of main memory.

3. C. Athanasiadis, J. Rambau, and F. Santos, "The Generalized Baues Problem for cyclic polytopes II", Publications De l'Institut Mathematique, Belgrade 66 (1999) 3-15.

4. M. Azaola and F. Santos, "The number of triangulations of the cyclic polytope $C(n, n-4)$ ", Discrete Comput. Geom. 27 (2002) 29-48, Geometric combinatorics (San Francisco, CA/Davis, CA, 2000).

5. H. J. Baues, "Geometry of loop spaces and the cobar construction", Memoirs of the American Mathematical Society 25 (1980) 1-171.

6. L. J. Billera, M. M. Kapranov, and B. Sturmfels, "Cellular strings on polytopes", Proceedings of the American Mathematical Society 122 (1994) 549-555.

7. L. J. Billera and B. Sturmfels, "Fiber polytopes", Annals of Mathematics 135 (1992) 527-549.

8. A. Björner, "Topological methods", in Handbook of Combinatorics, R. L. Graham, M. Grötschel, and L. Lovász, eds., North Holland, Amsterdam, 1995, 1819-1872.

9. A. Björner, M. Las Vergnas, B. Sturmfels, N. White, and G. M. Ziegler, Oriented matroids, second ed., Encyclopedia of Mathematics and its Applications, vol. 46, Cambridge University Press, Cambridge, 1999.

10. M. de Berg, O. Cheong, M. van Kreveld, and M. Overmars, Computational Geometry: Algorithms and Applications, 3rd ed., Springer, 2008.

11. J. De Loera, J. Rambau, and F. Santos, Triangulations - Structures for Applications and Algorithms, Algorithms and Computation in Mathematics, vol. 25, Springer, 2010.

12. P. Dehornoy, "Tamari lattices and the symmetric Thompson monoid", in this volume, 2011.

13. T. K. Dey, "On counting triangulations in $d$ dimensions", Comput. Geom. 3 (1993) 315-325.

14. P. Edelman, V. Reiner, and J. Rambau, "On subdivision posets of cyclic polytopes", European Journal of Combinatorics 21 (2000) 85-101.

15. P. H. Edelman and V. Reiner, "The higher Stasheff-Tamari posets", Mathematika 43 (1996) 127-154.

16. H. Edelsbrunner, Geometry and topology for mesh generation, Cambridge Monographs on Applied and Computational Mathematics, vol. 7, Cambridge University Press, Cambridge, 2001.

17. H. Edelsbrunner and N. R. Shah, "Incremental topological flipping works for regular triangulations", in Proceedings of the 8th annual ACM Symposium on Computational Geometry, ACM press, 1992, 43-52.

18. S. Felsner and H. Weil, "A theorem on higher Bruhat orders", Discrete \& Computational Geometry 23 (2000) 121-127.

19. I. M. Gelfand, M. M. Kapranov, and A. V. Zelevinsky, "Discriminants of polynomials in several variables and triangulations of Newton polyhedra", Leningrad Mathematical Journal 2 (1991) 449-505. 
20. , Discriminants, Resultants, and Multidimensional Determinants, Mathematics: Theory \& Applications, Birkhäuser, Boston, 1994.

21. C. Hohlweg, "Permutahedra and associahedra: Generalized associahedra from the geometry of finite reflection groups", in this volume, 2011.

22. S. Huang and D. Tamari, "Problems of associativity: A simple proof for the lattice property vof systems ordered by a semi-associative law", J. Combinatorial Theory Ser. A 13 (1972) 7-13.

23. D. Huguet and D. Tamari, "La structure polyédrale des complexes de parenthésages", J. Combin. Inform. System Sci. 3 (1978) 69-81.

24. M. M. Kapranov and V. A. Voevodsky, "Combinatorial-geometric aspects of polycategory theory: pasting schemes and higher Bruhat orders (list of results)", Cahiers de Topologie et Géométrie différentielle catégoriques 32 (1991) 11-27.

25. Y. I. Manin and V. V. Schechtman, "Arrangements of hyperplanes, higher braid groups and higher Bruhat orders", Advanced Studies in Pure Mathematics 17 (1989) 289-308.

26. S. Oppermann and H. Thomas, "Higher dimensional cluster combinatorics and representation theory", math arXiv (2010) ??

27. U. Pachner, "P.L. homeomorphic manifolds are equivalent by elementary shellings", European J. Combin. 12 (1991) 129-145.

28. J. M. Pallo, "An algorithm to compute the Möbius function of the rotation lattice of binary trees”, RAIRO Inform. Théor. Appl. 27 (1993) 341-348.

29. J. Rambau, Projections of Polytopes and Polyhedral Subdivisions, Berichte aus der Mathematik, Shaker, Aachen, 1996, Dissertation, TU Berlin.

30. __ "A suspension lemma for bounded posets", J. Combin. Theory Ser. A 80 (1997) 374-379.

31. _ "Triangulations of cyclic polytopes and higher Bruhat orders", Mathematika 44 (1997) $162-194$.

32. __ "TOPCOM: Triangulations of point configurations and oriented matroids", in Mathematical Software-ICMS 2002, A. M. Cohen, X.-S. Gao, and N. Takayama, eds., World Scientific, 2002, 330-340.

33. J. Rambau and F. Santos, "The Generalized Baues Problem for cyclic polytopes I", European Journal of Combinatorics 21 (2000) 65-83.

34. J. Rambau and G. M. Ziegler, "Projections of polytopes and the Generalized Baues Conjecture", Discrete \& Computational Geometry 16 (1996) 215-237.

35. N. Reading, "From the Tamari lattice to Cambrian lattices and beyond", in this volume, 2011.

36. V. Reiner, "The generalized Baues problem", in New Perspectives in Algebraic Combinatorics (Berkeley, CA, 1996-97), Math. Sci. Res. Inst. Publ., vol. 38, Cambridge Univ. Press, Cambridge, 1999, 293-336.

37. J. Richter-Gebert and G. M. Ziegler, "Zonotopal tilings and the Bohne-Dress theorem", in Proceedings "Jerusalem Combinatorics '93", H. Barcelo and G. Kalai, eds., Contemporary Mathematics, vol. 178, American Mathematical Society, 1994, 211-232.

38. F. Santos, "A point configuration whose space of triangulations is disconnected", Journal of the American Mathematical Society 13 (2000) 611-637.

39. __ "Non-connected toric Hilbert schemes", Mathematische Annalen 332 (2005) 645-665.

40. D. D. Sleator, R. E. Tarjan, and W. P. Thurston, "Rotation distance, triangulations, and hyperbolic geometry", Journal of the American Mathematical Society 1 (1988) 647-681.

41. J. D. Stasheff, "How i 'met' Dov Tamari", in this volume, 2011.

42. J. R. Stembridge, "A Maple package for posets", Free software, available online, 2008.

43. H. Thomas, "New combinatorial descriptions of the triangulations of cyclic polytopes and the second higher Stasheff-Tamari posets", Order 19 (2002) 327-342.

44. "Maps between higher Bruhat orders and higher Stasheff-Tamari posets", in Formal Power Series and Algebraic Combinatorics Conference - Linköping, Sweden, 2003, 2003.

45. _ "The Tamari lattice as it arises in quiver representations", in this volume, 2011.

46. G. M. Ziegler, "Higher Bruhat orders and cyclic hyperplane arrangements", Topology 32 (1993) 259-279.

47. G. M. Ziegler, Lectures on polytopes, Graduate Texts in Mathematics, vol. 152, Springer-Verlag, New York, 1995 